\title{
Modelling the influence of spatially varying hydrodynamics on the cross-sectional stability of double inlet systems
}

\author{
Ronald L. Brouwer · Henk M. Schuttelaars • \\ Pieter C. Roos
}

Received: 14 April 2013 / Accepted: 25 September 2013 / Published online: 25 October 2013

(c) Springer-Verlag Berlin Heidelberg 2013

\begin{abstract}
The cross-sectional stability of double inlet systems is investigated using an exploratory model that combines Escoffier's stability concept for the evolution of the inlet's cross-sectional area with a two-dimensional, depthaveraged (2DH) hydrodynamic model for tidal flow. The model geometry consists of four rectangular compartments, each with a uniform depth, associated with the ocean, tidal inlets and basin. The water motion, forced by an incoming Kelvin wave at the ocean's open boundary and satisfying the linear shallow water equations on the $f$-plane with linearised bottom friction, is in each compartment written as a superposition of eigenmodes, i.e. Kelvin and Poincaré waves. A collocation method is employed to satisfy boundary and matching conditions. The analysis of resulting equilibrium configurations is done using flow diagrams.

Model results show that internally generated spatial variations in the water motion are essential for the existence of
\end{abstract}

Responsible Editor: Rockwell Geyer

This article is part of the Topical Collection on Physics of Estuaries and Coastal Seas 2012

R. L. Brouwer $(\triangle)$

Section of Coastal Engineering, Faculty of Civil Engineering and Geosciences, Delft University of Technology, P.O. Box 5048, 2600 GA, Delft, The Netherlands

e-mail: r.l.brouwer@tudelft.nl

\section{H. M. Schuttelaars}

Department of Applied Mathematical Analysis,

Faculty of Electrical Engineering, Mathematics and Computer

Science, Delft University of Technology, P.O. Box 5031,

2600 GA, Delft, The Netherlands

\section{P. C. Roos}

Department of Water Engineering and Management, Faculty of Engineering Technology, University of Twente, P.O. Box 217, 7500 AE, Enschede, The Netherlands stable equilibria with two inlets open. In the hydrodynamic model used in the paper, both radiation damping into the ocean and basin depth effects result in these necessary spatial variations. Coriolis effects trigger an asymmetry in the stable equilibrium cross-sectional areas of the inlets. Furthermore, square basin geometries generally correspond to significantly larger equilibrium values of the inlet crosssections. These model outcomes result from a competition between a destabilising (caused by inlet bottom friction) and a stabilising mechanism (caused by spatially varying local pressure gradients over the inlets).

Keywords Cross-sectional stability $\cdot$ Double inlet system · Equilibrium · Idealised morphodynamic modelling $\cdot$ Tidal hydrodynamics

\section{Introduction}

About $10 \%$ of the world's coastline consists of barrier coasts (Glaeser 1978). They generally display a chain of barrier islands separated by tidal inlets that connect one or more back-barrier basins to an ocean or a coastal sea. The combination of tidal inlet and back-barrier basin is commonly known as a tidal inlet system. Examples are found along the Wadden Sea coast of the Netherlands, Germany and Denmark (see Fig. 1, left panel), the Ría Formosa in southern Portugal and the U.S. east coast. These morphologically dynamic systems are subject to potentially conflicting interests of economy, coastal safety and ecology.

To manage these different interests, it is important to understand the long-term morphological evolution of these inlet systems and their adaptation to natural changes (e.g. sea level rise, storm-induced barrier island breaching) and 
Fig. 1 Left panel Satellite image of the Dutch, German and Danish Wadden Sea coast (Copyright: Common Wadden Sea Secretariat). Right panel Western Dutch Wadden Sea coast and associated tidal inlet systems (Copyright: USGS/ESA)
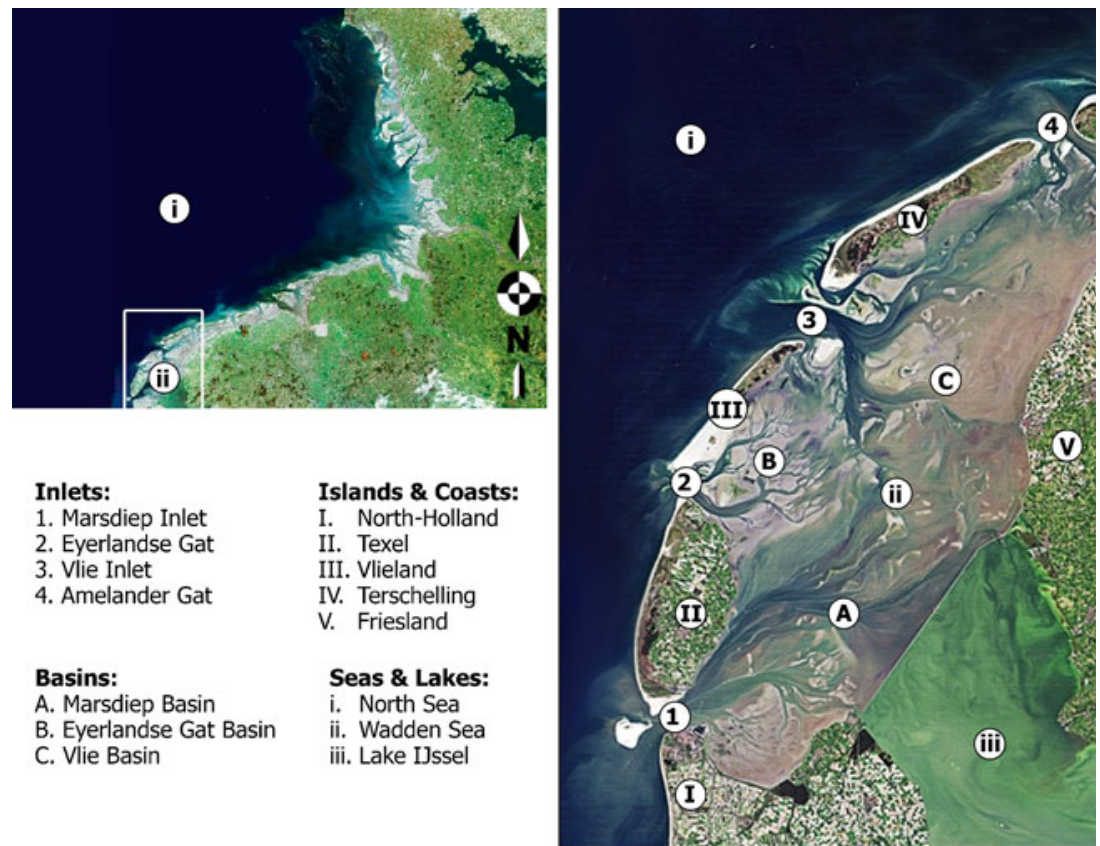

human interventions (e.g. basin reduction, dredging activities, inlet relocation). For example, the closure of the Zuiderzee (now called Lake IJssel) in the north-west of the Netherlands in 1932 induced extensive morphological changes in the Dutch Wadden Sea that are still felt today (for more details, see, e.g. Elias et al. 2003). An example of barrier island breaching occurred recently (29 October 2012) along the south shore of Long Island (NY) where Hurricane Sandy created three breaches on Fire Island, which led to (expensive) measures to close them again (National Park Service 2012).

The stability properties of these systems can be inferred from the morphological evolution of the cross-sectional area of the tidal inlets. For single inlet systems in equilibrium, various empirical relationships exist between the inlet's cross-sectional area $A$ and ebb-tidal prism $P$ (e.g. LeConte 1905; O'Brien 1931; Jarrett 1976). Alternatively, Escoffier (1940) related the size of an inlet and its stability to the relative strength of two opposing forces:"on the one hand wind and waves that are continuously pushing sand into the inlet and on the other hand tidal currents which carry sand out of the channel back into the sea or back-barrier basin." Based on this balance, he proposed an equilibrium condition $U=U_{\text {eq }}$ for the flow velocity amplitude $U$ in the inlet. If $U>U_{\text {eq }}$, the inlet's cross-sectional area increases (erodes), and if $U<U_{\text {eq }}$, it decreases (accretes). An equilibrium is considered stable when after being perturbed, the crosssectional area returns to its original equilibrium value. To calculate $U$, Escoffier used a simple hydrodynamic model (Brown 1928), that assumes a uniformly fluctuating surface elevation in the basin, and prescribed a sinusoidal forcing at the seaside. It was found that, in general, one stable and one unstable equilibrium cross-sectional area exist.

Van de Kreeke (1985, 1990a, b) extended the above stability concept to double inlet systems, taking the Pass Cavallo-Matagorda Inlet system on the US Gulf coast as an example. Another example of a double inlet system is the Marsdiep-Vlie system on the Dutch Wadden Sea coast as depicted in Fig. 1, right panel. For these systems, an equilibrium is considered stable when, after being perturbed, the cross-sectional area of both inlets return to their original equilibrium values. Similarly to Escoffier (1940), in his model schematisation, the surface elevation in the basin was assumed to fluctuate uniformly. Considering a balance between bottom friction in the inlets and the pressure gradient over the inlets, he concluded that only unstable equilibria exist, i.e. one inlet closes and the other remains open. This model result contradicts observations in, for instance, the Dutch Wadden Sea (Louters and Gerritsen 1994) and the Portuguese Ría Formosa (Salles et al. 2005; Pacheco et al. 2008) where tidal inlet systems with more than one inlet connecting the back-barrier basin to the ocean are stable on a historical time scale ( $\sim$ centuries). This inconsistency between model results and observations may be attributed to the too-restrictive assumption of a uniformly fluctuating basin surface elevation: Escoffier (1977) already suggested that spatial variations in basin surface elevation may lead to stable configurations with more than one inlet open. Previous studies showed that accounting for these spatial variations explicitly (van de Kreeke et al. 2008; de Swart and Volp 2012), by implementing a topographic high in the basin schematisation, or parametrically (Brouwer et al. 
2012a), by including entrance/exit losses in the dynamic equation, stable equilibrium configurations with more than one inlet open could be found. However, in these studies, the (sub-)basin surface elevation was still assumed to fluctuate uniformly, neglecting effects of basin depth and geometry. In addition, the forcing off the inlets was prescribed, thereby neglecting the influence of the inlet system on the hydrodynamics on the seaward side of the tidal inlets: radiation damping. This mechanism can be explained by considering an incoming tidal wave that induces an oscillatory flow in each inlet, and consequently triggering co-oscillations in the basin. These co-oscillations result in waves radiating away into the ocean, influencing the surface elevations off the inlets. Finally, Coriolis effects have been omitted so far, which are also likely to influence the surface elevations in the ocean, inlets and basin.

To scrutinise Escoffier's (1977) suggestion, this study aims to investigate the influence of a spatially varying water motion on the cross-sectional stability of double inlet systems. In particular, we examine individual contributions of basin depth effects, radiation damping and Coriolis effects. Therefore, Escoffier's concept of inlet stability is combined with a two-dimensional, depth-averaged (2DH) hydrodynamic model (see Taylor 1920; Godin 1965; Roos and Schuttelaars 2011; Roos et al. 2011) based on the linear shallow water equations in a rotating, idealised geometry consisting of ocean, tidal inlets and basin.

This study is organised as follows. In the following section, we present the new 2DH model and solution method. Subsequently, in Section 3, the water motion for a rotating double inlet system is discussed, as well as the influence of radiation damping, basin depth effects and Coriolis effects on the cross-sectional stability of double inlet systems. In addition, the effect of different basin geometries is examined. Finally, Sections 4 and 5 contain the discussion and conclusions, respectively.

\section{Model and method}

\subsection{Cross-sectional stability}

Following Escoffier (1940), an inlet is assumed to be in equilibrium if the amplitude of the cross-sectionally averaged inlet velocity $U_{p}(p=1,2)$ is equal to the so-called equilibrium velocity $U_{\text {eq. }}$. Assuming a negligible sediment exchange between the inlets and basin, alternative to Escoffier (1940), this equilibrium velocity is defined as the amplitude of the ebb-tidal current that is just sufficient to flush the sediment carried into the inlet by waves and flood currents (van de Kreeke 1992; de Swart and Zimmerman 2009). Hence, inlets are in equilibrium (assuming average weather conditions as opposed to storm conditions) if there is a balance between the volume of sediment entering the inlet during flood and leaving the inlet during ebb. The import of sediment $M$ is defined as a constant fraction of the littoral drift, while the export of sediment is taken proportional to a power of the cross-sectionally averaged ebb-tidal velocity amplitude $U_{p}$. We assume that the inlet accretes uniformly over its entire length if the difference between the amount of sediment that enters and leaves the inlet during a tidal cycle is positive. Similarly, the inlet erodes uniformly if this difference is negative. Under these assumptions, the rate of change of the cross-sectional area of inlet $p$ can be written as (van de Kreeke 1998)

$\frac{d A_{p}}{d t}=\frac{M}{L_{p}}\left[\left(\frac{U_{p}}{U_{\mathrm{eq}}}\right)^{n}-1\right], \quad p=1,2$.

Here, $A_{p}$ is the cross-sectional area of inlet $p$ (square metres); $t$ is time (seconds); $L_{p}$ is the length of inlet $p$ (metres) (in this study, they are assumed to be equal, i.e. $\left.L_{1}=L_{2}\right) ; M$ is the sediment import (a constant fraction of the littoral drift) (cubic metres per year); $U_{p}$ is the crosssectionally averaged ebb-tidal velocity amplitude of inlet $p$ (metres per second); $U_{\text {eq }}$ is the equilibrium velocity (metres per second); and $n$ is a power whose value depends on the adopted sand-transport law. Here, $n$ is assumed to be 3 . The value of $U_{\mathrm{eq}}$ is usually taken as $1 \mathrm{~m} \mathrm{~s}^{-1}$ (Bruun 1978; see Brouwer et al. (2012a) for a brief discussion of the range of $U_{\text {eq }}$ values used in literature); it depends on the littoral drift, sediment characteristics, wave climate and tidal period (van de Kreeke 1992). Here, we take the parameters $L_{p}, M$ and, hence, $U_{\text {eq }}$ to be identical for both inlets. If $U_{p}=U_{\text {eq }}$, it follows from Eq. 1 that $d A_{p} / d t=0$. If this is true for both inlets, the inlet system is in equilibrium. The equilibrium is stable when both cross-sectional areas return to their original equilibrium values after a small perturbation.

\subsection{Hydrodynamic model formulation}

As discussed in the previous section, the sediment transport mechanisms differ between flood and ebb: during flood, the presence of both waves (seaward of the tidal inlet) and tidal currents contribute to the sediment transport into the tidal inlet, while during ebb, only tidal currents result in sediment transport out of the inlet (assuming the influence of waves to be negligible in the tidal inlet and basin). Since the processes seaward of the tidal inlet are not modelled explicitly, the sediment transport $M$ during flood is modelled parametrically, proportional to $U_{\text {eq }}^{3}$. This difference in sediment transport during flood and ebb results in tide residual sediment transport and, using Eq. 1, evolution of the cross-sectional area of the inlets. This concept enables us to calculate the evolution of a double inlet system in a systematic and computationally efficient way, which is essential 
in view of the large temporal and spatial scales involved and the desire to conduct extensive sensitivity analyses. In turn, this facilitates the discovery of important dependencies and identification of physical mechanisms. In this study, we calculate the inlet flow velocities $U_{p}$ using an idealised 2DH hydrodynamic model based on the modelling approach described in Taylor (1920), Godin (1965), Roos and Schuttelaars (2011) and Roos et al. (2011). Using this hydrodynamic model, the influence of some of the assumptions made in the description of the hydrodynamics in the more classical lumped parameter (LP) models (see, e.g. van de Kreeke 2004; Brouwer et al. 2012a) on the crosssectional stability of tidal inlets can be investigated. The model extensions, listed in Table 1, are the following:

1. Since the adjacent sea/ocean is contained in the model geometry, the tidal wave travelling past the inlet system is part of the solution. This implies that the amplitude and phase differences between the two inlets are not prescribed externally but follow from the model.

2. The depth of the ocean, inlets and basin are taken into account explicitly, thus influencing both tide propagation and tide dissipation in each element.

3. By adopting a two-dimensional model, the ocean, inlets and basin each have a length and width, allowing for a systematic study into the influence of geometries on inlet stability.

4. Coriolis effects are taken into account.

The model calculates tidal elevation and flow in a geometry consisting of four adjacent rectangular compartments

Table 1 Comparison of classical LP model and 2DH model

\begin{tabular}{lll}
\hline Aspect & LP model & 2DH model \\
\hline Inertia & Yes & Yes \\
Inlet depth & Yes & Yes \\
Basin depth & No & Yes \\
Ocean depth & No & Yes \\
Entrance/exit losses & Yes & No \\
Radiation damping & No & Yes \\
Coriolis effects & No & Yes \\
Forcing & Prescribed & Dynamic result \\
Basin geometry & Surface area & Explicit \\
\hline
\end{tabular}

${ }^{a}$ Possibility to include parametrically (see Maas 1997)

${ }^{\mathrm{b}}$ The ocean surface elevation is prescribed externally just outside the inlets

'The model is forced by an incoming Kelvin wave through the open boundary of the ocean compartment. The ocean surface elevation just outside the inlets is a result of the dynamics equations

${ }^{\mathrm{d}}$ Basin is schematised as a basin area only. Hypsometric effects can be included (see de Swart and Volp 2012)

${ }^{\mathrm{e}}$ Basin has a specific length, width and depth constituting a double inlet system (see Fig. 2). It consists of four compartments of length $L_{k}$, width $B_{k}$ and (uniform) depth $H_{k}(k=0, \ldots, 3)$. Compartment 0 , which has an open boundary at $x=-L_{0}$, represents the ocean/sea. Compartment 1 and 2 are two inlet channels of rectangular cross-section and equal length $\left(L_{1}=L_{2}\right)$. Compartment 3 is the tidal basin. The double inlet system (Compartments 1 , 2 and 3 ) is symmetrically aligned with respect to the central axis $y_{0}$ of the ocean compartment. As a result, $y_{3}=y_{0}$ and $\left|y_{1}-y_{0}\right|=\left|y_{2}-y_{0}\right|$. Furthermore, the distance between the inlets' central axes is defined as $\Delta y=y_{2}-y_{1}$. In each compartment, conservation of momentum and mass is expressed by the linearised depth-averaged shallow water equations on the $f$-plane:

$$
\begin{aligned}
& \frac{\partial u_{k}}{\partial t}-f v_{k}+\frac{r_{k} u_{k}}{H_{k}}=-g \frac{\partial \zeta_{k}}{\partial x}, \\
& \frac{\partial v_{k}}{\partial t}+f u_{k}+\frac{r_{k} v_{k}}{H_{k}}=-g \frac{\partial \zeta_{k}}{\partial y}, \\
& \frac{\partial \zeta_{k}}{\partial t}+H_{k}\left[\frac{\partial u_{k}}{\partial x}+\frac{\partial v_{k}}{\partial y}\right]=0 .
\end{aligned}
$$

For compartment $k, u_{k}(x, y, t)$ and $v_{k}(x, y, t)$ are the depth-averaged flow velocity components in positive $x$-and $y$-direction, respectively, and $\zeta_{k}(x, y, t)$ is the free surface elevation. Furthermore, $g=9.81 \mathrm{~m} \mathrm{~s}^{-2}$ is the gravitational acceleration and $f=2 \Omega \sin \vartheta$ is the Coriolis parameter in the northern Hemisphere $(f>0)$, with $\Omega=7.292 \times 10^{-5}$ $\operatorname{rad~s}^{-1}$ the angular frequency of the Earth's rotation and $\vartheta$ the central latitude of the system. In Eqs. 2-4, $r_{k}$ is a linear bottom friction coefficient:

$r_{k}=\frac{8 c_{D} U_{k}}{3 \pi}$.

It is obtained from Lorentz' linearisation of a quadratic friction law (Lorentz 1926; Zimmerman 1982) with a default value of the drag coefficient $c_{D}=2.5 \times 10^{-3}$ and typical flow velocity scale $U_{k}$. Since velocities will differ per compartment, each compartment is allowed to have a different bottom friction coefficient $r_{k}$. Calculating each friction coefficient thus requires an estimate of the flow velocity scale, which is defined as the velocity amplitude averaged over compartment $k$. The friction coefficients are obtained using an iterative procedure (for details, see Appendix A.4).

At the closed boundaries, a no-normal flow condition is imposed $(u=0$ or $v=0)$. Across the interfaces between ocean and inlets, and between inlets and basin, continuity of elevation and normal flux is required:

$\begin{array}{ll}\zeta_{0}=\zeta_{p}, & H_{0} u_{0}=H_{p} u_{p}, \\ \zeta_{p}=\zeta_{3}, & H_{p} u_{p}=H_{3} u_{3},\end{array}$$$
\text { with } p=1,2 \text {. Analogous to the classical Taylor (1920) }
$$
problem, the system is forced by a single, sinusoidal 
Fig. 2 Schematisation of the model geometry

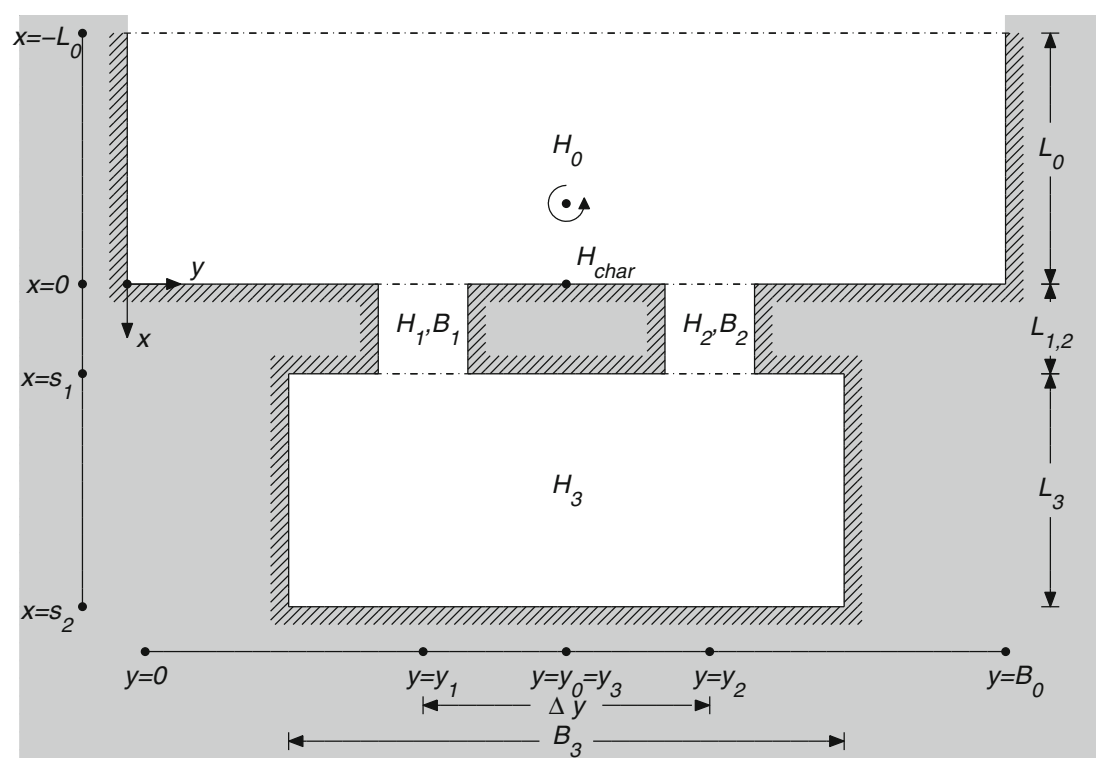

incoming Kelvin wave with angular frequency $\omega$ and elevation amplitude $Z_{i n c}$ entering through the open boundary of the ocean compartment. Due to the Coriolis effect, the Kelvin wave travels along the coast past the two inlets, thus forcing the flow in the double inlet system. The reflected Kelvin wave, along with other waves generated within the model domain, leave the ocean compartment without reflection.

To solve the problem for $\zeta_{k}, u_{k}$ and $v_{k}$, the solution in each compartment is written as a truncated sum of analytical wave solutions in an infinite channel. Subsequently, a collocation technique is used to satisfy the no-normal flow condition at the closed boundaries as well as the matching conditions (cf. Eqs. 6 and 7). For a detailed description of the solution method, the reader is referred to Appendix A.1-A.3.

\subsection{Flow diagram}

To determine the equilibrium cross-sectional areas and their stability properties, we show the results using so-called flow diagrams. A flow diagram plots the cross-sectional area of Inlet $1, A_{1}$, against that of Inlet $2, A_{2}$, and consists of the equilibrium flow curves for both inlets together with a vector plot. The equilibrium flow curve for Inlet 1 represent the locus of $\left(A_{1}, A_{2}\right)$ values for which for that inlet $U_{1}\left(A_{1}, A_{2}\right)=U_{\text {eq }}$ and similarly for inlet 2, i.e. $U_{2}\left(A_{1}, A_{2}\right)=U_{\text {eq }}$. Here, the velocities $U_{1}$ and $U_{2}$ are the cross-sectionally averaged velocity amplitudes, calculated using the hydrodynamic equations, described above, at the transect $x=s_{1} / 2$, i.e. halfway between the basin and the sea, for different combinations of $\left(A_{1}, A_{2}\right)$. During the calculation of $U_{1}$ and $U_{2}$ for different combinations of
$\left(A_{1}, A_{2}\right)$, the inlet's cross-sectional areas $A_{p}$ are assumed to remain geometrically similar (O'Brien and Dean 1972). Assumed is a rectangular cross-section with a depth to width ratio $H_{p} / W_{p}=0.005$ for both inlets. Hence, the shape factor $\gamma_{p}=\sqrt{H_{p} / W_{p}} \approx 0.07$ is constant for all values of the cross-sectional areas. The intersections of the $U_{p}=U_{\mathrm{eq}^{-}}$ curves represent sets of equilibrium cross-sectional areas. To determine the stability of the equilibria, vectors are added to the flow diagram. These vectors are the unit vectors in the direction of $\left(d A_{1} / d t, d A_{2} / d t\right)$ calculated from Eq. 1 . The vectors indicate the system's tendency to evolve, i.e. the direction in which the values of cross-sectional areas change when they are not in equilibrium. For a detailed description of the flow diagram, the reader is referred to van de Kreeke et al. (2008).

\section{Model results}

The influence of the various model extensions, compared to the LP model, on the water motion and equilibrium configuration of a double inlet system will be presented in this section. Default values for the geometry and parameters used in this section are given in Table 2. These values are based on characteristic values of the Marsdiep-Vlie system in the western Dutch Wadden Sea found in literature and using Google Earth. Even though previous studies (e.g. Roos and Schuttelaars 2011; Roos et al. 2011) using a similar model approach showed that sea surface elevations at various locations can be well reproduced, it is emphasised that the aim is neither to accurately reproduce the water motion of the Marsdiep-Vlie system, nor to predict its long-term evolution. 
Table 2 Parameter values for the default case (see also Fig. 4)

\begin{tabular}{lll}
\hline Parameter & Symbol & Value \\
\hline Compartments & & \\
Ocean length $(\mathrm{km})$ & $L_{0}$ & 50 \\
Ocean width $(\mathrm{km})$ & $B_{0}$ & 200 \\
Ocean depth $(\mathrm{m})$ & $H_{0}$ & 20 \\
Inlet lengths $(\mathrm{km})$ & $L_{1}=L_{2}$ & 6 \\
Inlet widths $(\mathrm{km})$ & $B_{1}=B_{2}$ & 3 \\
Inlet depths $(\mathrm{m})$ & $H_{1}=H_{2}$ & 15 \\
Basin length $(\mathrm{km})$ & $L_{3}$ & 30 \\
Basin width $(\mathrm{km})$ & $B_{3}$ & 40 \\
Basin depth $(\mathrm{m})$ & $H_{3}$ & 5 \\
General & & \\
Central latitude $\left({ }^{\circ} \mathrm{N}\right)$ & $\vartheta$ & 53 \\
Char. tidal amplitude $(\mathrm{m})$ & $Z_{\text {char }}$ & 0.8 \\
Tidal frequency $\left(\mathrm{rad} \mathrm{s}^{-1}\right)$ & $\omega$ & $1.4 \times 10^{-4}$ \\
Sediment import $\left(\mathrm{m}^{3} \mathrm{yr}^{-1}\right)$ & $M$ & $5 \times 10^{5}$ \\
Equilibrium velocity $\left(\mathrm{m} \mathrm{s}^{-1}\right)$ & $U_{\text {eq }}$ & 1 \\
Inlet shape factor $(-)$ & $\gamma_{p}^{2}$ & $5 \times 10^{-3}$ \\
\hline
\end{tabular}

The Marsdiep-Vlie system is situated at a latitude of $53^{\circ} \mathrm{N}$ (see Fig. 1, right panel). In the north-east, a typical topographic high separates the back-barrier basin of this system from the tidal basin of the Amelander Gat. To the east, the coast of Friesland forms a natural boundary. A long barrier that separates Lake IJssel from the Wadden sea creates a boundary to the south. The basin drained by the Eyerlandse Gat is small and is separated from the Marsdiep and Vlie basins by a very long and shallow tidal divide. It can be considered as an independent inlet system, which hardly influences the water motion in the Marsdiep-Vlie system (Zimmerman 1976). To roughly represent the basin surface area of the Marsdiep-Vlie system, a value of $L_{3} \times B_{3}=30 \times 40=1,200 \mathrm{~km}^{2}$ is adopted (approximated using Google Earth), with uniform depth $H_{3}=5 \mathrm{~m}$ (e.g. Dastgheib et al. 2008). The inlet compartments have identical dimensions $L_{1,2} \times B_{1,2}=6 \times 3 \mathrm{~km}^{2}$ (Google Earth) and reference depths $H_{1,2}=15 \mathrm{~m}$. The inlet spacing is chosen to be $\Delta y=10 \mathrm{~km}$, so that different basin geometries can be adopted with the same inlet spacing. The width of the ocean compartment is chosen as $B_{0}=200 \mathrm{~km}$, such that the tidal wave in front of the inlets closely resembles a Kelvin wave. Furthermore, an arbitrary ocean length $L_{0}$ of $50 \mathrm{~km}$ and an ocean depth $H_{0}$ of $20 \mathrm{~m}$ is assumed to be representative for the North Sea.

The tide in the area is dominated by the semi-diurnal lunar constituent (M2) with radian frequency $\omega=1.4 \times$ $10^{-4} \mathrm{~s}^{-1}$. The associated characteristic tidal amplitude for the Marsdiep-Vlie system, $Z_{\text {char }}=0.8 \mathrm{~m}$, is defined on the coastline exactly between the two inlets (i.e. at $(x, y)=$ $\left(0, B_{0} / 2\right)$ in Fig. 2). This value equals the mean tidal amplitude, i.e. averaged over a spring-neap cycle, in front of the Marsdiep, $Z_{\text {Mars }}=0.7 \mathrm{~m}$, and Vlie Inlet, $Z_{\text {Vlie }}=$ $0.9 \mathrm{~m}$ (e.g. Beets et al. 1992). To subsequently determine the typical amplitude $Z_{\text {inc }}$ of the incoming Kelvin wave (see Section 2), the model is run with the ocean compartment only, as if there were no double inlet system connected to it. Consequently, depending on ocean depth and latitude, $Z_{\text {inc }}$ can be determined by trial and error so that $Z_{\text {char }}=0.8 \mathrm{~m}$. The reason for defining a $Z_{\text {char }}$ is to make sure that the forcing of the double inlet system is approximately similar for each combination of parameter values. Hence, model results can be compared among each other.

\subsection{Water motion}

The water motion for the default case is shown in Fig. 3. It displays the calculated instantaneous surface elevations and flow velocities at four moments of the tidal cycle. Left panels show the double inlet system with the ocean compartment and right panels only show the double inlet system, i.e. inlets and basin. The ebb phase is indicated by cold colours and the flood phase by warm colours. Notice that the velocity scale in Fig. $3 b$, $f$ is different from the other figure sub-panels.

Figure 3 shows that surface elevations in ocean, inlets and basin display significant spatial variations in the order of magnitude of tens of centimetres. In the inlets, the spatial variation of the surface elevation is predominantly in the along-channel direction. Furthermore, it follows that the basin surface elevation constantly lags the ocean surface elevation, resulting in pressure gradients across the tidal inlets.

\subsection{Influence of radiation damping, basin depth effects and Coriolis effects}

The results of the previous section clearly show that spatial variations in surface elevation and horizontal velocities in both ocean and basin are significant. To systematically investigate their influence on the stability of double inlet systems, the various mechanisms that are included in our model formulation resulting in these spatial inhomogeneities will be investigated separately. As a first step, parameters in the 2DH model are chosen such that the characteristics of the LP model used in previous studies (e.g. van de Kreeke 1985, 1990a, b; van de Kreeke et al. 2008; Brouwer et al. 2012a) are reproduced. Next, additional mechanisms, such as radiation damping, basin depth effects and Coriolis effects, will be introduced one at a time, and their influence on the cross-sectional stability of double inlet systems will be investigated. 
Fig. 3 Instantaneous surface elevation and flow velocities in a rotating double inlet system connected to an ocean at four moments of the tidal cycle: $t=0$ (a and $\mathbf{b}), t=T / 4$ (c and d), $t=T / 2$ (e and f) and $t=3 T / 4$ (g and h). Here, $T=2 \pi / \omega=12.42 \mathrm{~h}$ denotes the tidal period. Surface elevations are in metres, arrows denote flow direction and magnitude, co-phase lines are depicted in white with intervals of $10^{\circ}$ and co-range contours are depicted in black with intervals of $0.05 \mathrm{~m}$. Default parameter values are listed in Table 2 a $\quad t=0$

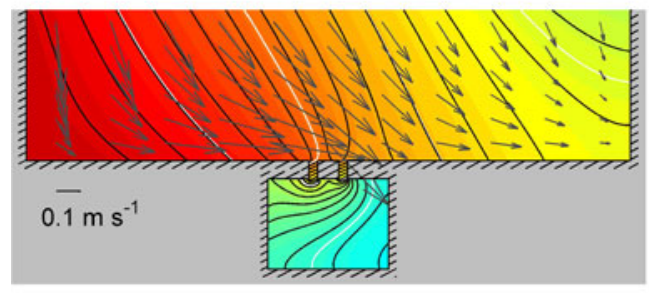

C $\mathrm{t}=\mathrm{T} / 4$

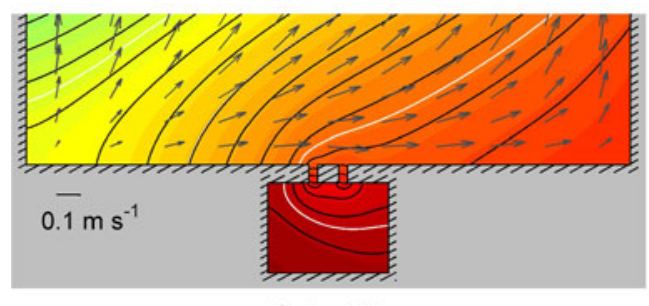

e $t=T / 2$

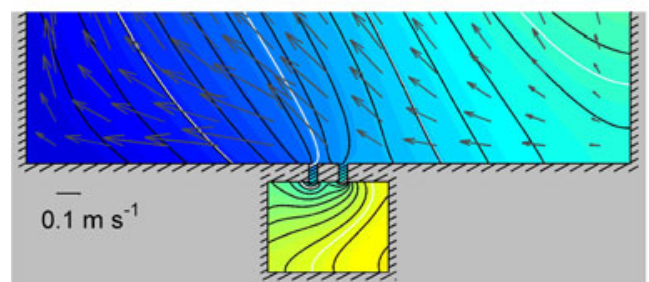

g $\mathrm{t}=3 \mathrm{~T} / 4$

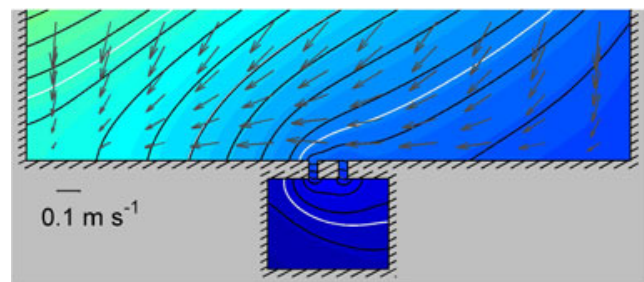

b $\quad t=0$

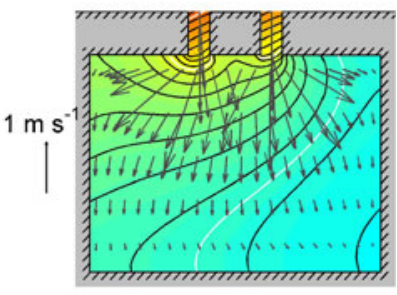

d $t=T / 4$

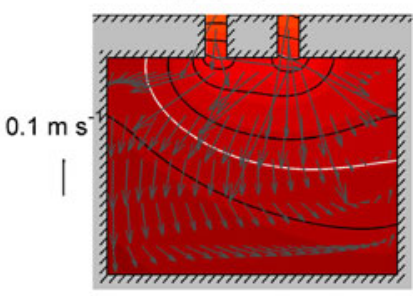

f $t=T / 2$

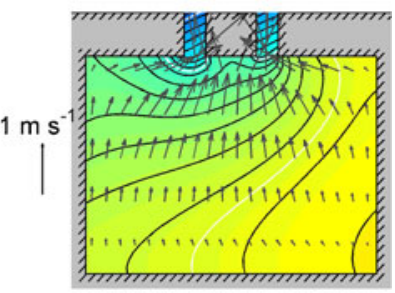

h $t=3 T / 4$
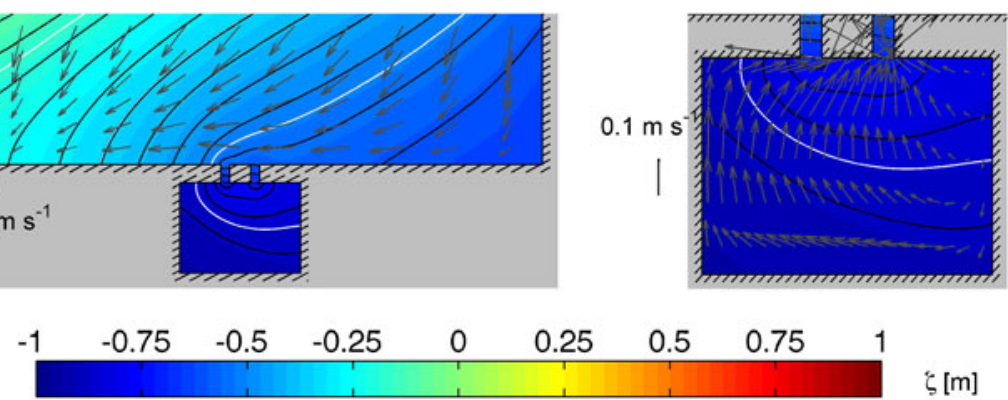

\subsubsection{Lumped parameter model limit}

To approximate the model formulation used in the LP models as closely as possible, the ocean and basin compartment are assumed to be deep, $H_{0}=H_{3}=1,000 \mathrm{~m}$. As a result, depth effects in the basin and ocean become negligible, and the incoming wave has neither an amplitude nor a phase difference between the two inlets. Additionally, the central latitude of the double inlet system is assumed to be located at the equator, i.e. $\vartheta=0$, thereby omitting Coriolis effects. Other parameter values used are listed in Table 2. The resulting surface elevation in ocean and basin are approximately uniform (see Fig. 4a). The corresponding flow diagram is presented in Fig. 4b.
The flow diagram shows a configuration without stable equilibria. Hence, ultimately only one inlet remains open and the other closes. Additional calculations (not shown here) with varying inlet lengths $L_{1}$ and $L_{2}$, inlet shape factor $\gamma$ and characteristic tidal amplitude $Z_{\text {char }}$, show that the equilibrium configuration might change in shape, but will not yield more than two equilibrium cross-sectional areas. Hence, no stable equilibria will arise. This result confirms the results by van de Kreeke (1990a): when only bottom friction in the inlets and pressure gradients over the inlets are retained and assuming a uniformly fluctuating basin surface elevation, at best two combinations of equilibrium cross-sectional areas exist that are both unstable. In addition, Fig. $4 \mathrm{~b}$ is in agreement with results of van de Kreeke et al. (2008) for large cross-sectional areas of the 


\section{a Surface elevation amplitudes for LP model limit}

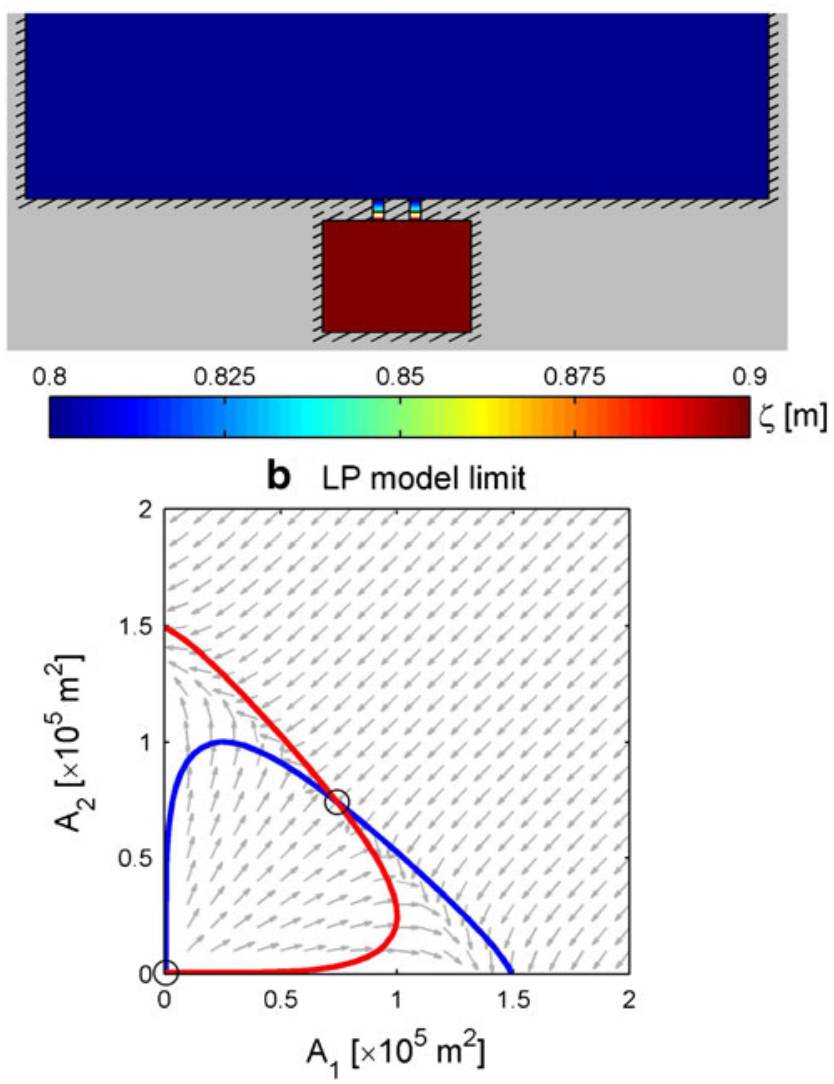

Fig. 4 a Surface elevation amplitudes for a double inlet system in the LP model limit. b Flow diagram for a double inlet system with inertia and inlet bottom friction only. The blue and red line represent the equilibrium flow curve for Inlet 1 and 2, respectively; the black circles indicate an unstable equilibrium; the grey arrows indicate the system's tendency when out of equilibrium. In these figures $H_{0}=$ $H_{3}=1,000 \mathrm{~m}, \vartheta=0$ and other parameter values are as listed in Table 2

topographic high and Brouwer et al. (2012a) for an entrance/exit loss coefficient $m=0$.

\subsubsection{Radiation damping}

As explained in the "Introduction," the mechanism of radiation damping influences the surface elevation of each inlet by waves radiating away into the ocean. Since its effect on the surface elevations in the ocean is proportional to the ratio between inlet and ocean depth (see, e.g. Eq. 4.2 in Buchwald 1971), its influence on the water motion and the cross-sectional stability of double inlet systems can be investigated by considering three different ocean depths: $H_{0}=20 \mathrm{~m}, H_{0}=30 \mathrm{~m}$ and $H_{0}=40 \mathrm{~m}$. The basin depth and the central latitude are chosen equal to the LP model limit, i.e. $H_{3}=1,000 \mathrm{~m}$ and $\vartheta=0$, respectively. Using these parameter values, the surface elevation in the basin is approximately uniform and (symmetric) inhomogeneities in the water motion are observed in the ocean (see Fig. 5a). The flow diagrams for ocean depths $H_{0}=20 \mathrm{~m}, H_{0}=30 \mathrm{~m}$ and $H_{0}=40 \mathrm{~m}$ are shown in sub-panels b, c and d of Fig. 5, respectively.

Figure $5 \mathrm{~b}$ shows that for $H_{0}=20 \mathrm{~m}$, a stable equilibrium exists; there are four equilibrium cross-sectional areas, one of which is stable. Hence, there is an equilibrium situation where both inlets are open. Increasing $H_{0}$ to $30 \mathrm{~m}$ (Fig. 5c), the stable equilibrium still exists, but the two unstable equilibria (excluding the one near the origin) have moved considerably closer to the stable equilibrium. Eventually, for $H_{0}=40 \mathrm{~m}$ (Fig. 5d), the stable equilibrium has disappeared (in fact, has merged with the two unstable equilibria) and only two unstable equilibrium cross-sectional areas remain; ultimately, one of the inlets will close while the other remains open. In addition, from the flow diagrams in Fig. 5, it follows that the set of stable equilibrium cross-sectional areas slightly decrease from $\left(A_{1}^{\mathrm{eq}}, A_{2}^{\mathrm{eq}}\right) \approx\left(0.81 \times 10^{5}, 0.81 \times 10^{5}\right)$ for $H_{0}=20 \mathrm{~m}$ to $\left(A_{1}^{\mathrm{eq}}, A_{2}^{\mathrm{eq}}\right) \approx\left(0.79 \times 10^{5}, 0.79 \times 10^{5}\right)$ for $H_{0}=30 \mathrm{~m}$ and disappears between $H_{0}=30 \mathrm{~m}$ and $H_{0}=40 \mathrm{~m}$. The gradual disappearance of the stable equilibrium in Fig. 5b, c to $5 \mathrm{~d}$ implies that for increasing ocean depth, the radiation damping mechanism becomes weaker. Additional calculations without bottom friction in the ocean $c_{D}=0$ in compartment 0 , not shown here) resulted in qualitatively similar results as Fig. 5, indicating that the presence of the stable equilibrium in Fig. 5 is due to the radiation damping mechanism only.

Besides the ratio between inlet and ocean depth, also the inlet length determines the strength of the radiation damping mechanism (see, e.g. Maas 1997; Miles 1948; Garrett 1975). It turns out that the radiation damping mechanism is inversely proportional to the inlet length. Hence, this contribution loses importance with increasing inlet length. This sensitivity is shown in Fig. 6, which shows flow diagrams for inlet lengths $L_{1}=L_{2}=6 \mathrm{~km}$ (Fig. 6a), $L_{1}=L_{2}=12 \mathrm{~km}$ (Fig. 6b) and $L_{1}=L_{2}=24 \mathrm{~km}$ (Fig. 6c). The ocean depth is taken to be $20 \mathrm{~m}$. These figures show that the stable equilibrium disappears for an inlet length between $L_{1}=L_{2}=12 \mathrm{~km}$ (Fig. 6b) and $L_{1}=L_{2}=24 \mathrm{~km}$ (Fig. 6c). Furthermore, the stable equilibrium cross-sectional areas increase for increasing inlet lengths from $\left(A_{1}^{\mathrm{eq}}, A_{2}^{\mathrm{eq}}\right) \approx\left(0.81 \times 10^{5}, 0.81 \times 10^{5}\right)$ for $L_{1}=$ $L_{2}=6 \mathrm{~km}$ to $\left(A_{1}^{\mathrm{eq}}, A_{2}^{\mathrm{eq}}\right) \approx\left(0.89 \times 10^{5}, 0.89 \times 10^{5}\right)$ for $L_{1}=L_{2}=12 \mathrm{~km}$.

\subsubsection{Basin depth effects}

The effect of basin depth on the cross-sectional stability of double inlet systems is investigated by considering three basin depths: $H_{3}=3 \mathrm{~m}, H_{3}=5 \mathrm{~m}$ and $H_{3}=10 \mathrm{~m}$. The ocean depth and the central latitude are still equal to the LP 
Fig. 5 a Surface elevation amplitudes for a double inlet system with radiation damping in the ocean, inertia and inlet bottom friction for $H_{0}=20 \mathrm{~m}$. Associated flow diagrams are depicted for $\mathbf{b} H_{0}=20 \mathrm{~m}, \mathbf{c}$ $H_{0}=30 \mathrm{~m}$ and $\mathbf{d} H_{0}=40 \mathrm{~m}$ Furthermore, $L_{1}=L_{2}=6 \mathrm{~km}$, $H_{3}=1,000 \mathrm{~m}, \vartheta=0$ and other parameter values are listed in Table 2. The blue and red line represent the equilibrium flow curve for Inlet 1 and 2, respectively; a black circle and asterisk indicates an unstable and stable equilibrium, respectively; and the grey arrows indicate the system's tendency when out of equilibrium a Surface elevation amplitudes for radiation damping only
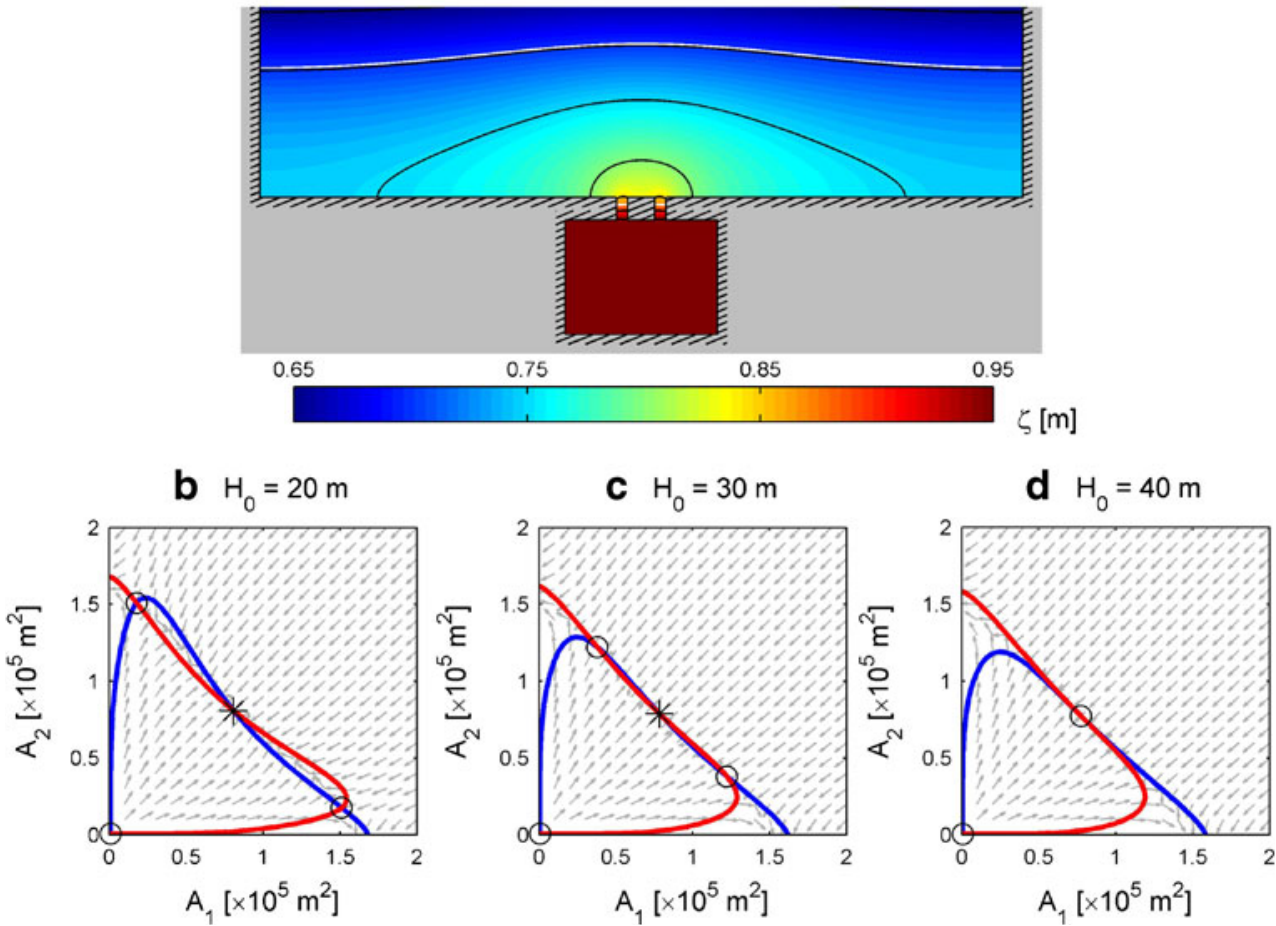

model limit, i.e. $H_{0}=1,000 \mathrm{~m}$ and $\vartheta=0$, respectively. Since only basin depth effects are considered, spatial variations in the water motion are observed inside the basin, whereas the surface elevation in the ocean is approximately uniform (see Fig. 7a). The flow diagrams corresponding to the different depths are shown in sub-panels $b, c$ and d of Fig. 7.

From these figures, it follows that basin depth effects trigger a stabilising mechanism. All flow diagrams in Fig. 7 show an equilibrium configuration with one stable equilibrium with two inlets open. For $\mathrm{H}_{3}=3 \mathrm{~m}, \mathrm{H}_{3}=5 \mathrm{~m}$ and $H_{3}=10 \mathrm{~m}$, the stable set of equilibrium cross-sectional areas $\left(A_{1}^{\mathrm{eq}}, A_{2}^{\mathrm{eq}}\right)$ is approximately $\left(0.47 \times 10^{5}, 0.47 \times 10^{5}\right)$, $\left(0.79 \times 10^{5}, 0.79 \times 10^{5}\right)$ and $\left(0.80 \times 10^{5}, 0.80 \times 10^{5}\right)$, respectively. This shows that stable equilibrium crosssections are smaller for shallower basins.

From the model Eqs. 2-5, it follows that varying basin depth affects both tide propagation and tide dissipation (the latter also through the velocity scale in our Lorentz linearisation). In other simulations, not reported here, we have varied the drag coefficient $c_{D}$ and found that, for a fixed basin depth, the equilibrium values of $A_{1}$ and $A_{2}$ decrease for increasing $c_{D}$ values.

\subsubsection{Coriolis effects and LP model limit}

The last mechanism that is considered concerning the crosssectional stability of double inlet systems is related to the

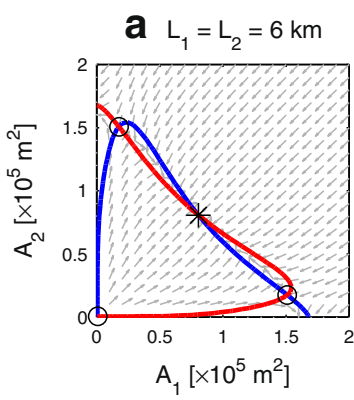

Fig. 6 Flow diagrams for a double inlet system with radiation damping in the ocean, inertia and inlet bottom friction for a $L_{1}=L_{2}=6$ $\mathrm{km}$, b $L_{1}=L_{2}=12 \mathrm{~km}$ and $\mathbf{c} L_{1}=L_{2}=24 \mathrm{~km}$. Furthermore, b $\mathrm{L}_{1}=\mathrm{L}_{2}=12 \mathrm{~km}$
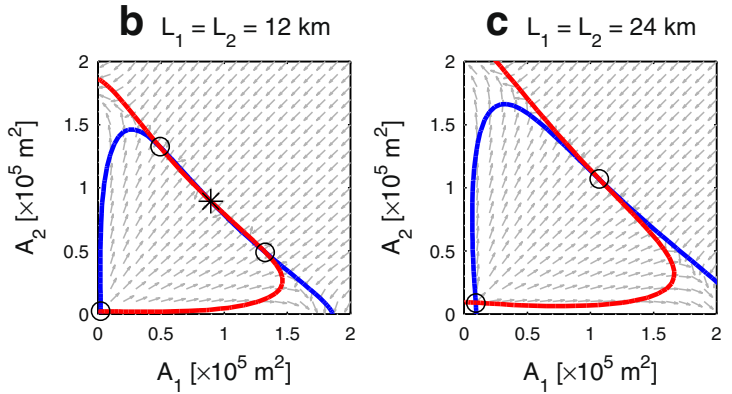

$H_{0}=20 \mathrm{~m}, H_{3}=1,000 \mathrm{~m}, \vartheta=0$ and other parameter values are listed in Table 2. For a description of the figures see the caption of Fig. 5 
Fig. 7 a Surface elevation amplitudes for a double inlet system with basin depth effects, inertia and inlet bottom friction for $H_{3}=5 \mathrm{~m}$. Associated flow diagrams are depicted for $\mathbf{b}$ $H_{3}=3 \mathrm{~m}, \mathbf{c} H_{3}=5 \mathrm{~m}$ and $\mathbf{d}$ $H_{3}=10 \mathrm{~m}$. Furthermore, $H_{0}=1,000 \mathrm{~m}, L_{1}=L_{2}=6$ $\mathrm{km}, \vartheta=0$ and other parameter values are listed in Table 2. For a description of the figures see the caption of Fig. 5 a Surface elevation amplitudes for basin depth effects only
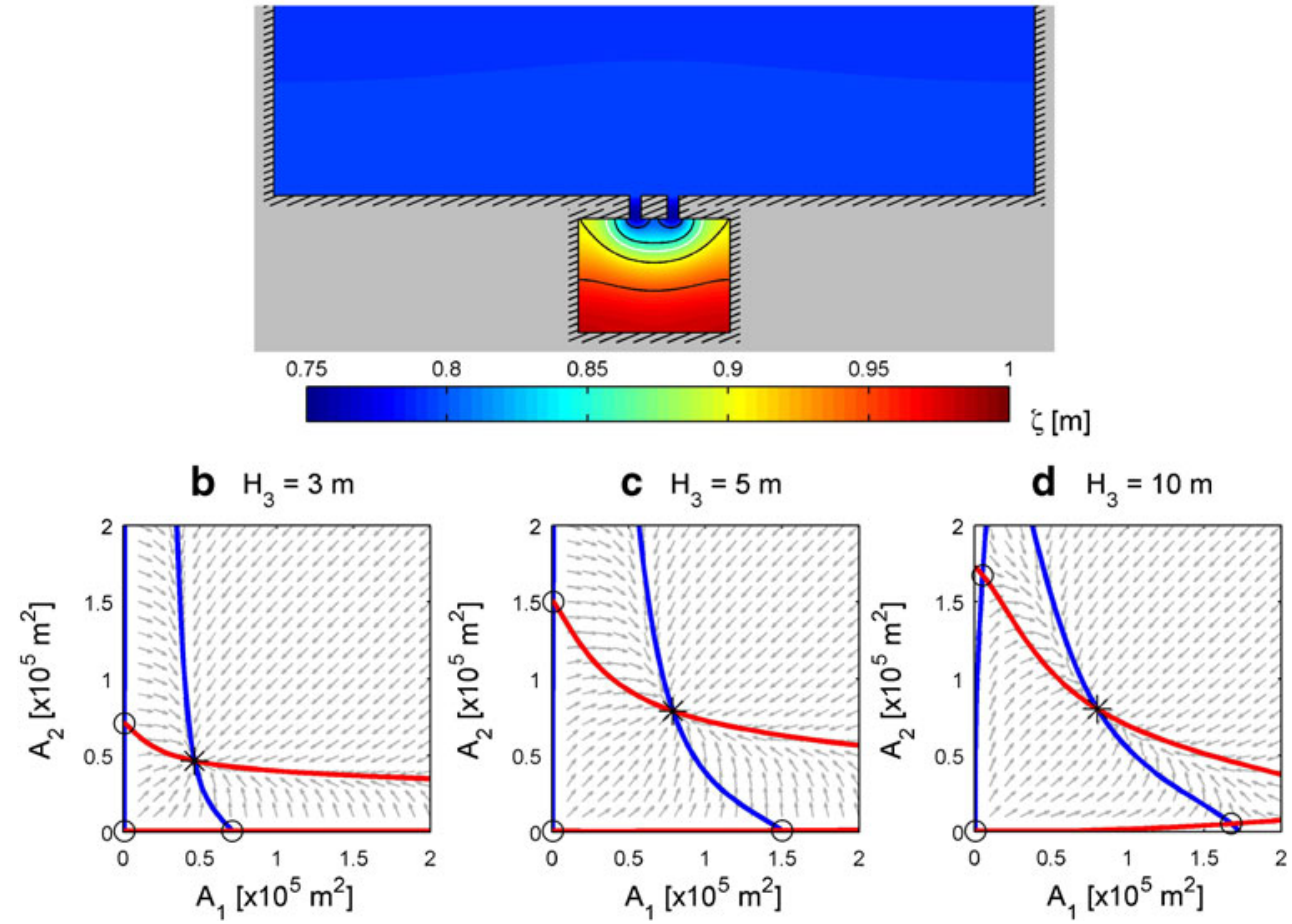

inclusion of Coriolis effects. So far, the influence of Coriolis effects on cross-sectional stability of double inlet systems has not been studied. They mainly influence the flow characteristics in ocean and basin and as a result influence the spatial structure of surface elevations. When, in addition to inertia and inlet bottom friction (LP model limit), only Coriolis effects are included by increasing the central latitude to a (North Sea) value of $\vartheta=53^{\circ} \mathrm{N}$, calculations show (not depicted here) the same equilibrium configuration as when the double inlet system is situated on the equator (see Fig. 4b). This can also be expected as the Rossby radius of deformation, $L_{R}=\sqrt{g H} / f$, is large for a large depth $H$ and small Coriolis parameter $f$ and therefore Coriolis effects only will not affect the equilibrium configuration for the parameter values chosen here.

\subsubsection{Coriolis effects and all mechanisms}

The previous section demonstrated that Coriolis effects in combination with the LP model limit will not affect the equilibrium configuration of a double inlet system. However, in the LP model limit, the ocean and basin depth are assumed to be relatively deep, i.e. $H_{0}=H_{3}=1,000 \mathrm{~m}$, respectively. When these depths are reduced to realistic values, Coriolis effects may affect the equilibrium configuration. Therefore, in this section, all mechanisms discussed previously are taken into account, i.e. Coriolis effects, radiation damping and basin depth effects. The ocean and basin depths are assumed to be $H_{0}=20 \mathrm{~m}$ and $H_{3}=5 \mathrm{~m}$, respectively. Three cases are considered, one where the central latitude of the double inlet system is on the equator, $\vartheta=0$, one on $\vartheta=25^{\circ} \mathrm{N}$ and one on the latitude of the Marsdiep-Vlie system, $\vartheta=53^{\circ} \mathrm{N}$. The corresponding flow diagrams are depicted in Fig. 8a, b and c, respectively.

Figure 8 shows that, qualitatively, Coriolis effects do not change the presence of a stable equilibrium configuration. However, quantitatively Coriolis effects introduce an asymmetry in the equilibrium cross-sections. On the equator, $\vartheta=0$ (Fig. 8a), the set of equilibrium cross-sections $\left(A_{1}^{\mathrm{eq}}, A_{2}^{\mathrm{eq}}\right) \approx\left(0.81 \times 10^{5}, 0.81 \times 10^{5}\right)$; for $\vartheta=25^{\circ} \mathrm{N}$ (Fig. 8b), $\left(A_{1}^{\mathrm{eq}}, A_{2}^{\mathrm{eq}}\right) \approx\left(0.79 \times 10^{5}, 0.80 \times 10^{5}\right)$; and for $\vartheta=53^{\circ} N$ (Fig. $\left.8 \mathrm{c}\right),\left(A_{1}^{\mathrm{eq}}, A_{2}^{\mathrm{eq}}\right) \approx\left(0.75 \times 10^{5}, 0.79 \times 10^{5}\right)$. It follows that the smallest stable equilibrium cross-section is observed where the tide arrives first. In the case discussed here (on the northern hemisphere), the cross-sectional area of Inlet 1 is smaller than the one of Inlet 2 .

In addition, when considering a double inlet system situated on the equator $(\vartheta=0)$, the relative importance of radiation damping and basin depth effects on the stable equilibrium cross-sectional areas might be assessed by comparing Figs. 5b (radiation damping only), Fig. 7c (basin depth effects only) and Fig. 8a (radiation damping and basin depth effects). The corresponding values of the equilibrium crosssectional areas are $\left(A_{1}^{\mathrm{eq}}, A_{2}^{\mathrm{eq}}\right) \approx\left(0.81 \times 10^{5}, 0.81 \times 10^{5}\right)$, $\left(A_{1}^{\mathrm{eq}}, A_{2}^{\mathrm{eq}}\right) \approx\left(0.79 \times 10^{5}, 0.79 \times 10^{5}\right)$ and $\left(A_{1}^{\mathrm{eq}}, A_{2}^{\mathrm{eq}}\right) \approx$ $\left(0.81 \times 10^{5}, 0.81 \times 10^{5}\right)$, respectively. From these values, it is difficult to assess if either radiation damping or basin depth effects are dominant in determining the value 
Fig. 8 Flow diagrams for a double inlet system with radiation damping, basin depth effects, Coriolis effects, inertia and inlet bottom friction for $\mathbf{a}$ $\vartheta=0, \mathbf{b} \vartheta=25^{\circ} \mathrm{N}$ and $\mathbf{c}$ $\vartheta=53^{\circ} \mathrm{N}$. Other parameter values are listed in Table 2. For a description of the figures see the caption of Fig. 5
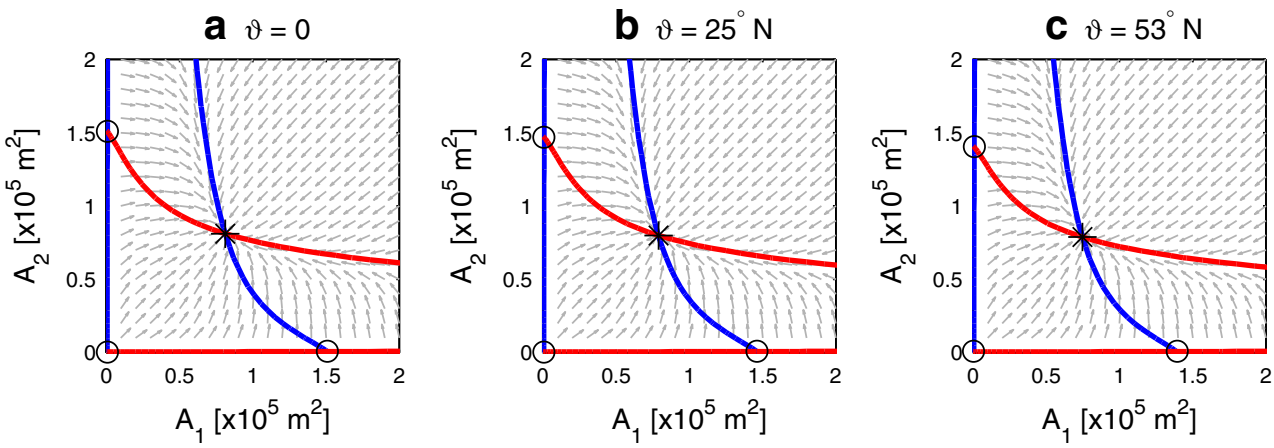

of the stable equilibrium cross-sectional area. However, the small difference in appearance between Figs. $7 \mathrm{c}$ and $8 \mathrm{a}$ after adding radiation damping suggest that basin depth effects are the more dominant mechanism in determining the equilibrium configuration of a double inlet system.

\subsection{Influence of basin geometry on cross-sectional inlet stability}

In the previous sections, we investigated the influence of radiation damping, basin depth effects and Coriolis effects on the cross-sectional stability of double inlet systems. In calculating the associated flow diagrams to study their influence, a basin geometry of $L_{3} \times B_{3}=30 \times 40 \mathrm{~km}^{2}$ was assumed. Observations show that basin geometries vary between short and wide basins and long and narrow ones. In this section, the influence of these different geometries on the cross-sectional stability is investigated. To that end, default parameter values denoted in Table 2 are used, but three different basin geometries $\left(L_{3} \times B_{3}\right)$ are chosen with a constant basin surface area of $1,200 \mathrm{~km}^{2}: 15 \times 80 \mathrm{~km}^{2}$, $30 \times 40 \mathrm{~km}^{2}$ (default case) and $60 \times 20 \mathrm{~km}^{2}$. For each calculation, the centre line of the tidal basin is on the same position as the centre line of the ocean. The inlet channels are $10 \mathrm{~km}$ apart and symmetrically positioned with respect to the centre lines of the ocean and basin. The resulting flow diagrams for each basin geometry are depicted in Fig. 9.

From Fig. 9, it follows that a single stable equilibrium exists for all three cases. In that sense, the basin geometry does not qualitatively change the presence of a stable equilibrium configuration. Quantitatively, however, basin geometry has a large effect on the equilibrium values of the cross-sectional areas. The largest equilibrium values are found for the $30 \times 40 \mathrm{~km}^{2}$ geometry in Fig. $9 \mathrm{~b}$, i.e. $\left(A_{1}, A_{2}\right) \approx\left(0.75 \times 10^{5}, 0.79 \times 10^{5}\right)$. The other basin shapes lead to significantly smaller equilibrium values (Fig. 9a, c): $\left(A_{1}, A_{2}\right) \approx\left(0.70 \times 10^{5}, 0.72 \times 10^{5}\right)$ for the $15 \times 80 \mathrm{~km}^{2}$ geometry and $\left(A_{1}, A_{2}\right) \approx\left(0.45 \times 10^{5}, 0.46 \times 10^{5}\right)$ for the $60 \times 20 \mathrm{~km}^{2}$ geometry, respectively. This leads to a difference in stable equilibrium cross-sectional areas up to a factor of approximately 2 , depending on the geometry of the basin.

\section{Discussion}

\subsection{Physical mechanisms}

Results of a recent model study by Roos et al. (2013), explaining observations on barrier island length in multiple inlet systems, revealed a physical explanation for the (non-)existence of stable double inlet systems with two inlets open. Similarly to our model, they use an exploratory model that combines Escoffier's stability concept with a hydrodynamic model for depth-averaged tidal flow in the inlets, basin and ocean. However, to systematically analyse the evolution of multiple inlet systems towards equilibrium states and to identify the underlying physical mechanisms, they solve the tidal flow pattern analytically. Hence, different to our study, they assume a single, representative value for the tidal flow velocity in the inlets and neglect bottom friction in the ocean and Coriolis effects throughout the entire model domain.

Their explanation is based on a competition between a destabilising and a stabilising mechanism. The first one is associated with bottom friction in the inlets. Because bottom friction depends on depth and therefore on the inlet's cross-sectional area, perturbations from the equilibrium cross-sectional area tend to amplify, thus acting as a destabilising mechanism. The second mechanism concerns the system's feedback to the local pressure gradients over the inlets. As it turns out, these local pressure gradients counteract the destabilising flow response from bottom friction alone, thus acting as a stabilising mechanism. Furthermore, they concluded that for stable double (or multiple) inlet systems to exist, this pressure gradient induced stabilising mechanism requires surface elevations in basin and ocean to vary in space.

The results presented in this study support the above explanation for the (in)stability of double inlet systems with 
Fig. 9 Flow diagrams for a double inlet system with radiation damping, basin depth effects, Coriolis effects, inertia and inlet bottom friction for a $L_{3} \times B_{3}=15 \times 80 \mathrm{~km}^{2}, \mathbf{b}$ $L_{3} \times B_{3}=30 \times 40 \mathrm{~km}^{2}$ and $\mathbf{c}$ $L_{3} \times B_{3}=60 \times 20 \mathrm{~km}^{2}$. Other parameter values are listed in Table 2 . For clarity the basin geometries are depicted as well. For a description of the figures see the caption of Fig. 5
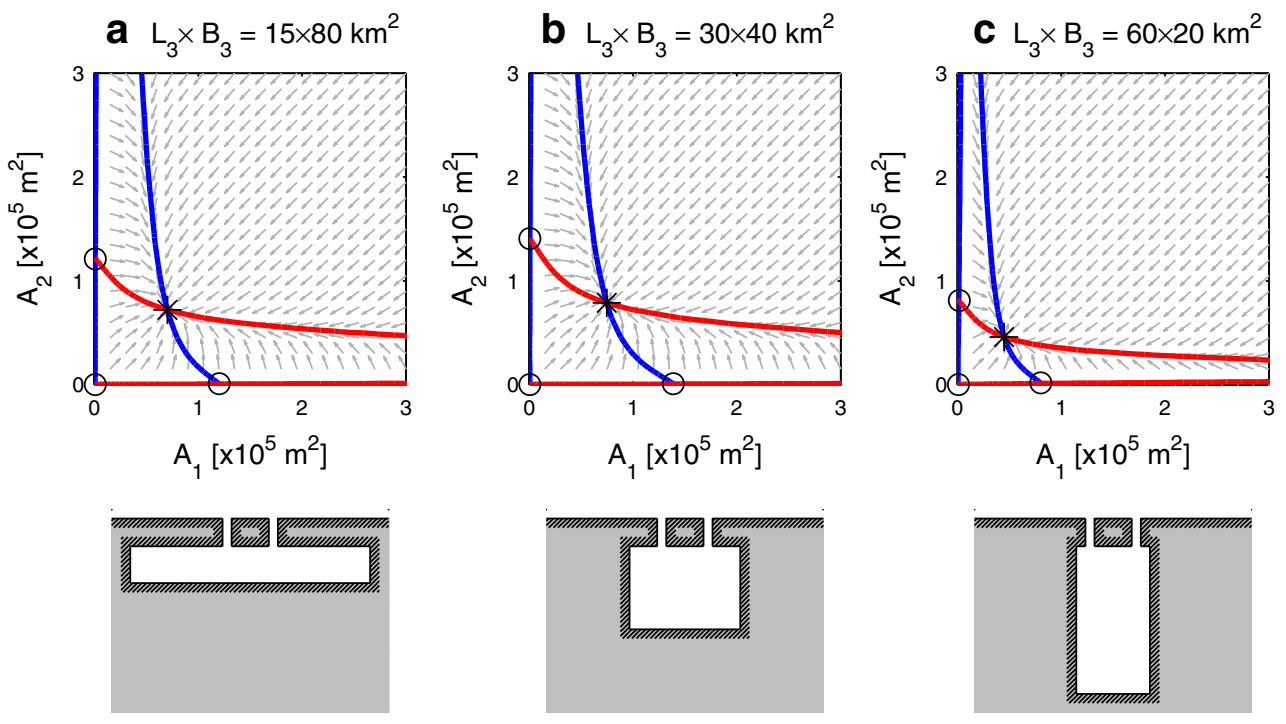

a model that includes spatially varying tidal flow, taking radiation damping, basin depth effects and Coriolis effects into account. Assuming a deep ocean and basin (LP model limit), hence retaining only bottom friction in the inlets, Fig. 4 showed that no stable equilibria with two inlets open exist. The same results were obtained by van de Kreeke (1990a) who used a LP model with essentially the same assumptions. According to Roos et al. (2013), the local pressure gradient over the inlets associated with a uniformly fluctuating basin surface elevation is not sufficient to counteract the destabilising flow response from inlet bottom friction alone.

When accounting for spatially varying surface elevations in ocean and/or basin, our results show that stable equilibrium configurations can be found. Figures 5 and 6 illustrated that, depending on ocean depth and inlet length, spatial variations in ocean surface elevation alone (radiation damping) can result in a stable double inlet system with two inlets open. Similarly, basin depth effects alone (Fig. 7) can lead to stable double inlet systems. Apparently, in these cases the stabilising mechanism, induced by local pressure gradients over the inlets, can overcome the destabilising effect from inlet bottom friction. Roos et al. (2013) explained the positive effect of spatially varying surface elevations on the stabilising mechanism by considering spatial variations in the basin only. As it turns out, spatial variations in surface elevation in the ocean have the same effect on the stability of double inlet systems.

Furthermore, our study is the first to investigate the effects of Coriolis on the stability of double inlet systems. Our results revealed that without spatial variations in ocean and basin surface elevations, Coriolis effects do not lead to stable equilibrium configurations. When spatially varying surface elevations are accounted for, Coriolis effects modify the spatial structure of the surface elevations thereby introducing an asymmetry between the equilibrium inlet cross-sectional areas of both inlets.

Finally, model results suggest that basin geometry has a significant quantitative effect on the equilibrium values of the inlet's cross-sectional areas: square tidal basins generally result in significantly larger stable equilibrium values. This can be interpreted from the basin's hydrodynamics and resonance properties. For instance, the short and wide basin in Fig. 9a results in a standing tidal wave with length scale $\frac{1}{2} B_{3}$ and the long and small basin in Fig. 9c results in a tidal wave with length $L_{3}$. Hence, depending on the basin shape, and also depth, the basin's hydrodynamics and resonance properties may result in higher or lower inlet peak flow velocities. Subsequently, Escoffier's stability concept relates higher inlet peak flow velocities to larger values of the stable equilibrium cross-sectional area and vice versa for lower peak flow velocities. Note, that the same reasoning holds for a decreasing (or increasing) value of the equilibrium flow velocity. Investigating the exact influence of basin shape and depth on its hydrodynamics and resonance properties and therefore the stable equilibrium values of the inlet's cross-sectional areas is part of an ongoing study.

\subsection{Implications and outlook}

The outcomes of our exploratory model study give general insights into the long-term behaviour of double inlet systems, without expecting quantitative accuracy. Even so, our model results show good qualitative and reasonable quantitative agreement with observations from e.g. the western Dutch Wadden Sea. When including Coriolis effects, basin depth effects and radiation damping, Fig. 8c showed that equilibrium cross-sectional areas for Inlets 1 and 2 are $A_{1} \approx 75 \times 10^{3} \mathrm{~m}^{2}$ and $A_{2} \approx 79 \times 10^{3} \mathrm{~m}^{2}$, respectively. In calculating these values, the tide arrives first at Inlet 1. 
Comparing these model results with observations (Dronkers 1998; van de Kreeke 1998) of the Marsdiep Inlet, $A_{1} \approx$ $60 \times 10^{3} \mathrm{~m}^{2}$, and Vlie Inlet, $A_{2} \approx 65 \times 10^{3} \mathrm{~m}^{2}$, shows that qualitatively the inlet where the tide arrives first (Marsdiep Inlet) indeed has a smaller cross-sectional area than the other inlet (Vlie Inlet). Quantitatively, our model results slightly overestimate the observed cross-sectional areas of the Marsdiep and Vlie Inlets. Part of this is due to the fact that the observed values correspond to the smallest measured cross-sections, whereas our values are an average over the total inlet length.

To arrive at improved predictive capability, more processes need to be taken into account in the current exploratory approach and their influence on inlet stability needs to be investigated. Possible extensions are the following: allowing inlets to migrate, including unerodible (Pleistocene) layers in the basin or on barrier islands, and a more advanced representation of wind waves, and hence, littoral drift in the stability concept.

\section{Conclusions}

In this study, we investigated the influence of a spatially varying water motion on the cross-sectional stability of double inlet systems. In particular, we examined the individual contributions of radiation damping in the ocean, basin depth effects and Coriolis effects for different basin geometries. To that extent, a new 2DH hydrodynamic model that explicitly accounts for spatially varying surface elevations in ocean and basin is combined with a stability concept for tidal inlets (Escoffier 1940). Because the model is computationally efficient by schematising ocean, inlets and basin to rectangular compartments with uniform depth, it allows us to assess the equilibrium configurations by using flow diagrams. Below, we draw conclusions from the main model results.

When, in addition to inlet bottom friction and inertia, radiation damping is considered, stable equilibrium configurations with two inlets open are possible. The influence of the radiation damping mechanism is stronger for increasing ratios of inlet and ocean depth and decreasing inlet length.

Similarly to radiation damping, basin depth effects alone lead to the existence of stable equilibrium configurations. This mechanism leads to larger stable inlet cross-sectional areas for increasing basin depth.

Coriolis effects do not qualitatively change the stable equilibrium configuration of double inlet systems. However, quantitatively, model results including radiation damping and basin depth effects show that Coriolis effects result in an asymmetry of the stable equilibrium cross-sections. The cross-section of the inlet where the tide arrives first is smaller than that of the other inlet.
Analysis of the previous three mechanisms suggests that basin depth effects is the most important one, implying that care should be taken of assuming a uniformly fluctuating sea surface elevation for the entire back-barrier basin when investigating the stability of double inlet systems.

Next to the mechanisms that result in spatial variations in surface elevation, the influence of basin geometry is considered. Model results suggest that, qualitatively the basin geometry does not change the presence of stable equilibria. Quantitatively, a square basin shape generally corresponds to significantly larger equilibrium values. Specifically, taking a basin surface area of $1,200 \mathrm{~km}^{2}$, equilibrium values can differ up to a factor of approximately 2 depending on basin shape.

Model results further support the presence of a competition between a destabilising mechanism, caused by inlet bottom friction, and a stabilising mechanism, caused by the local pressure gradient over the inlets, that explain the (non-)existence of stable double inlet systems with two inlets open. Besides spatial variations in surface elevation in the basin (Roos et al. 2013), model results in this study also identified that spatial variations in the ocean surface elevation are crucial for the strength of the stabilising mechanism.

Finally, care should be taken in applying the model to natural double inlet systems. This has mainly to do with schematising the basin bathymetry into a mean depth, whereas natural back-barrier basins display a complex network of channels and shoals. To overcome this discrepancy, the basin depth could be used as a tuning parameter by requiring the total energy dissipation during a tidal cycle of the natural basin to be equal to the schematised one.

Acknowledgments The authors thank Mick van der Wegen and an anonymous reviewer for their constructive comments.

\section{Appendix A: Solution method 2DH model for double inlet systems}

In this appendix, we present the solution method for the 2DH hydrodynamical model for double inlet system. This method is based on the model originally proposed by Roos and Schuttelaars (2011) and Roos et al. (2011) for largescale semi-enclosed rectangular basins with longitudinal and transverse topographic steps.

\section{A.1 Wave solutions in a channel of uniform depth}

As a first step, we solve the system of Eqs. 2-4 to find the (analytical) wave solutions in infinitely long, semienclosed, rotating channels with uniform width $B_{k}$ and 
uniform depth $H_{k}$, including bottom friction. These equations can be written in terms of the surface level elevation $\zeta_{k}$, also know as the Klein-Gordon equation:

$\left[\mathcal{L}^{2}+f^{2}\right] \frac{\partial \zeta_{k}}{\partial t}-g H_{k} \mathcal{L}\left[\frac{\partial^{2} \zeta_{k}}{\partial x^{2}}+\frac{\partial^{2} \zeta_{k}}{\partial y^{2}}\right]=0$,

with differential operator $\mathcal{L}=\partial / \partial t+r_{k} / H_{k}$. A description of the other parameters can be found in the main text of Section 2.2. This equation allows for solutions of the form

$\zeta_{k}=\Re\left\{\tilde{\zeta}_{k}(y) e^{i\left[k_{k} x-\omega t\right]}\right\}$.

where $\mathfrak{R}$ denotes the real part, $\omega$ is the angular frequency, $k_{k}$ is the wave number and $\tilde{\zeta}_{k}(y)$ the lateral structure for the surface elevation. Using Eq. 9, it follows from Eq. 8 that the wave solutions involve Kelvin modes propagating in the positive and negative $x$-direction, as well as two families of Poincaré modes, generated at the closed ends. Introducing $\Phi_{k}=\left(\zeta_{k}, u_{k}, v_{k}\right)$, to symbolically represent the system's state in each compartment, the analytical expressions of these wave solutions in positive $x$-direction are as follows:

$\Phi_{k, m}^{\oplus}(x, y, t)=\left(\begin{array}{c}\zeta_{k, m}^{\oplus} \\ u_{k, m}^{\oplus} \\ v_{k, m}^{\oplus}\end{array}\right)=\Re\left\{Z_{k, m}^{\prime}\left(\begin{array}{l}\tilde{\zeta}_{k, m}^{\oplus}(y) \\ \left.\tilde{u}_{k, m}^{\oplus}(y)\right) \\ \tilde{v}_{k, m}^{\oplus}(y)\end{array}\right) e^{\left(i\left[k_{k, m}^{\oplus} x-\omega t\right]\right)}\right\}$,

with amplitude factor $Z_{k, m}^{\prime}(\mathrm{m})$, wave number $k_{k, m}^{\oplus}$ and lateral structures $\tilde{\zeta}_{k, m}^{\oplus}(y), \tilde{u}_{k, m}^{\oplus}(y)$ and $\tilde{v}_{k, m}^{\oplus}(y)$. For the Kelvin mode $(m=0)$ propagating in the positive $x$-direction and defining $\tilde{\Phi}_{k, 0}^{\oplus}(y)=\left(\tilde{\zeta}_{k, 0}^{\oplus}(y), \tilde{u}_{k, 0}^{\oplus}(y), \tilde{v}_{k, 0}^{\oplus}(y)\right)$, the lateral structures read

$\tilde{\Phi}_{k, 0}^{\oplus}(y)=\left(\begin{array}{c}1 \\ \lambda_{k}^{-1} \sqrt{g / H_{k}} \\ 0\end{array}\right) e^{-\frac{y}{\lambda_{k} R_{k}}}$,

and the wave number is

$k_{k, 0}^{\oplus}=\lambda_{k} K_{k}$,

Here, the reference wave number $K_{k}$, the Rossby radius of deformation $R_{k}$ (both typical for a classical Kelvin wave without bottom friction) and a frictional correction factor $\lambda_{k}$ are given by

$$
K_{k}=\frac{\omega}{\sqrt{g H_{k}}}, \quad R_{k}=\frac{\sqrt{g H_{k}}}{f}, \quad \lambda_{k}=\sqrt{1-\frac{i r_{k}}{\omega H_{k}}},
$$

respectively.
The wave number and lateral structures of the $m$ th Poincaré mode $(m>0)$ propagating (if free) or decaying (if evanescent) in the positive $x$-direction are given by

$$
\begin{aligned}
k_{k, m}^{\oplus} & =\sqrt{\lambda_{k}^{2} K_{k}^{2}-\lambda_{k}^{-2} R_{k}^{-2}-\alpha_{m}^{2}}, \\
\tilde{\Phi}_{k, m}^{\oplus}(y) & =\left(\begin{array}{c}
\cos \left(\alpha_{m} y\right)-\frac{f k_{k, m}^{\oplus}}{\alpha_{m} \omega \lambda^{2}} \sin \left(\alpha_{m} y\right) \\
\frac{g k_{k, m}^{\oplus}}{\omega \lambda_{k}^{2}} \cos \left(\alpha_{m} y\right)-\frac{f}{\alpha_{m} \lambda^{2} H_{k}} \sin \left(\alpha_{m} y\right) \\
\frac{i \omega}{\alpha_{m} \lambda_{k}^{2} H_{k}}\left[\lambda_{k}^{2}-\frac{k_{k, m}^{\oplus 2}}{K_{k}^{2}}\right] \sin \left(\alpha_{m} y\right)
\end{array}\right),
\end{aligned}
$$

respectively, with $\alpha_{m}=m \pi / B_{k}$.

The modes propagating or decaying in the negative $x$ direction are defined analogous to Eq. 10, but now using a superscript $\ominus^{\ominus}$ instead of a ${ }^{\oplus}$. By symmetry, the two types of modes $\tilde{\Phi}_{k, m}^{\oplus}$ and $\tilde{\Phi}_{k, m}^{\ominus}$ satisfy the following relationships:

$\tilde{\Phi}_{k, m}^{\ominus}(y)=\left(\begin{array}{c}\tilde{\zeta}_{k, 0}^{\oplus}\left(B_{k}-y\right) \\ -\tilde{u}_{k, 0}^{\oplus}\left(B_{k}-y\right) \\ -\tilde{v}_{k, 0}^{\oplus}\left(B_{k}-y\right)\end{array}\right), \quad k_{k, m}^{\ominus}=-k_{k, m}^{\oplus}$.

\section{A.2 Superposition of wave solutions}

The next step is to write the solution in each compartment as a truncated sum of the analytical wave solutions in an infinite channel derived above. To that end, $\Phi_{k}=\left(\zeta_{k}, u_{k}, v_{k}\right)$ is first rewritten as

$\Phi_{k}(x, y, t)=\Re\left\{\hat{\Phi}_{k}(x, y) e^{-i \omega t}\right\}$,

where the vector $\hat{\Phi}_{k}=\left(\hat{\zeta}_{k}, \hat{u}_{k}, \hat{v}_{k}\right)$ contains the complex amplitudes of surface elevation, longitudinal and lateral flow velocity, respectively.

Compartment 0 is forced at the open boundary by an incoming Kelvin wave. Since, the Poincaré modes bound to the open end $\left(x=-L_{0}\right)$ are neglected, the only mode propagating or decaying in the positive $x$-direction is the incoming Kelvin wave, with coastal amplitude $Z_{i n c}$ and initial phase $\varphi$ (both defined at $x=0$ ). At the other interface $x=0$, Kelvin modes $(m=0)$ and Poincaré modes $(m=1,2, \ldots, M)$ are generated (i.e. reflected or transmitted). The solution for Compartment 0 can thus be written as

$$
\begin{aligned}
\hat{\Phi}_{0}(x, y)= & Z_{i n c} e^{i \varphi} \tilde{\Phi}_{0,0}^{\oplus}(y) e^{i k_{0,0}^{\oplus}\left(x-L_{0}\right)} \\
& +\sum_{m=0}^{M} a_{0, m}^{\ominus} \tilde{\Phi}_{0, m}^{\ominus}(y) e^{i k_{0, m}^{\ominus} x},
\end{aligned}
$$

with truncation number $M$.

For the other compartments, modes propagating (if free) or decaying (if evanescent) in positive and negative 
$x$-direction exist. For the inlet compartments $(k=1,2)$, this results in

$$
\begin{aligned}
\hat{\Phi}_{k}(x, y)=\sum_{m=0}^{M}[ & a_{k, m}^{\oplus} \tilde{\Phi}_{k, m}^{\oplus}(y) e^{i k_{k, m}^{\oplus} x} \\
& \left.+a_{k, m}^{\ominus} \tilde{\Phi}_{k, m}^{\ominus}(y) e^{i k_{k, m}^{\ominus}\left(x-s_{1}\right)}\right],
\end{aligned}
$$

and for the basin compartment $(k=3)$ in

$$
\begin{aligned}
\hat{\Phi}_{3}(x, y)=\sum_{m=0}^{M}[ & a_{3, m}^{\oplus} \tilde{\Phi}_{3, m}^{\oplus}(y) e^{i k_{3, m}^{\oplus}\left(x-s_{1}\right)} \\
& \left.+a_{3, m}^{\ominus} \tilde{\Phi}_{3, m}^{\ominus}(y) e^{i k_{3, m}^{\ominus}\left(x-s_{2}\right)}\right],
\end{aligned}
$$

To make sure that the coefficients $a_{k, m}^{\oplus}$ and $a_{k, m}^{\ominus}$ in Eqs. 19 and 20 are of the same order of magnitude as $Z_{i n c}$, the exponential functions in these equations have been normalised to unity at the interfaces $x=0, x=s_{1}$ and $x=s_{2}$ respectively.

\section{A.3 Collocation technique}

Since each of the individual wave solutions in Eqs. 18-20 satisfies the no-normal flow condition at the closed boundaries, so do the superpositions $\hat{\Phi}_{k}$. A collocation technique is used to also satisfy the no-normal flow condition at the closed ends and the matching conditions in Eqs. 6 and 7 (see Fig. 10).

Defining $M+1$ lateral points $y_{n}=n B / M$ for $n=$ $0,1, \ldots, M$, the following conditions are required for the closed ends at $x=0\left(\mathcal{B}_{u, 0}\right), x=s_{1}\left(\mathcal{B}_{u, p}\right)$ and $x=s_{2}$ $\left(\mathcal{B}_{u, 3}\right)$ :

$\hat{u}_{0}=0, \quad$ at $(x, y)=\left(0, y_{n}\right) \in \mathcal{B}_{u, 0}$

$\hat{u}_{3}=0, \quad$ at $\left\{\begin{array}{l}(x, y)=\left(s_{1}, y_{n}\right) \in \mathcal{B}_{u, p}, \\ (x, y)=\left(s_{2}, y_{n}\right) \in \mathcal{B}_{u, 3},\end{array}\right.$ for continuity of elevation across the topographic steps at $x=0\left(\mathcal{T}_{0, p}\right)$ and $x=s_{1}\left(\mathcal{T}_{p, 3}\right)$ :

$\hat{\zeta}_{0}=\hat{\zeta}_{p}$

$$
\text { at }(x, y)=\left(0, y_{n}\right) \in \mathcal{T}_{0, p},
$$

$\hat{\zeta}_{p}=\hat{\zeta}_{3}$

at $(x, y)=\left(s_{1}, y_{n}\right) \in \mathcal{T}_{p, 3}$,

and for continuity of flux across the topographic steps:

$$
\begin{aligned}
H_{0} \hat{u}_{0}=H_{p} \hat{u}_{p}, & \text { at }(x, y)=\left(0, y_{n}\right) \in \mathcal{T}_{0, p}, \\
H_{p} \hat{u}_{p}=H_{3} \hat{u}_{3}, & \text { at }(x, y)=\left(s_{1}, y_{n}\right) \in \mathcal{T}_{p, 3},
\end{aligned}
$$

for $p=1,2$. This leads to a linear system of equations for the coefficients $a_{k, m}^{\oplus}$ and $a_{k, m}^{\ominus}$, which is solved using standard techniques.

\section{A.4 Iterative procedure to calculate friction coefficients}

Calculating the bottom friction coefficients $r_{k}$ in each compartment using Eq. 5 requires a typical velocity scale $U_{k}$. This quantity is defined as the square root of the squared velocity amplitude, averaged over a compartment, i.e.

$U_{k}^{2}=\frac{1}{L_{k} B_{k}} \int_{0}^{L_{k}} \int_{0}^{B_{k}}\left(|\hat{u}|^{2}+|\hat{v}|^{2}\right) d x d y$.

Subsequently, an iterative procedure is adopted to arrive at the appropriate value for $r_{k}$. As first guess, $r_{j}$ is obtained from Eq. 5 using a typical velocity scale for each compartment. For the ocean compartment, the typical velocity of a classical Kelvin wave without friction is used: $U_{0}=$ $Z \sqrt{g / H_{0}}$ (Pedlosky 1987). For the inlet compartments, the equilibrium velocity as proposed by Escoffier (1940) of $U_{1,2}=U_{\text {eq }}=1 \mathrm{~m} \mathrm{~s}^{-1}$ is used. Because the averaged flow velocities in shallow basins are lower than in the inlets, $U_{3}=0$ is taken as initial guess for the basin. Next, these initial guesses are used to obtain a new solution for $U_{k}, U_{k}^{\text {new }}$,
Fig. 10 Collocation points used to satisfy no-normal flow at the compartments' closed ends (open circles) and continuity of elevation $\zeta$ and flux $\mathrm{Hu}$ across the topographic steps (solid circles). Combined sets of Kelvin/Poincaré modes are indicated by double arrows, pointing in the direction of propagation/decay; a single arrow represents the incoming Kelvin wave

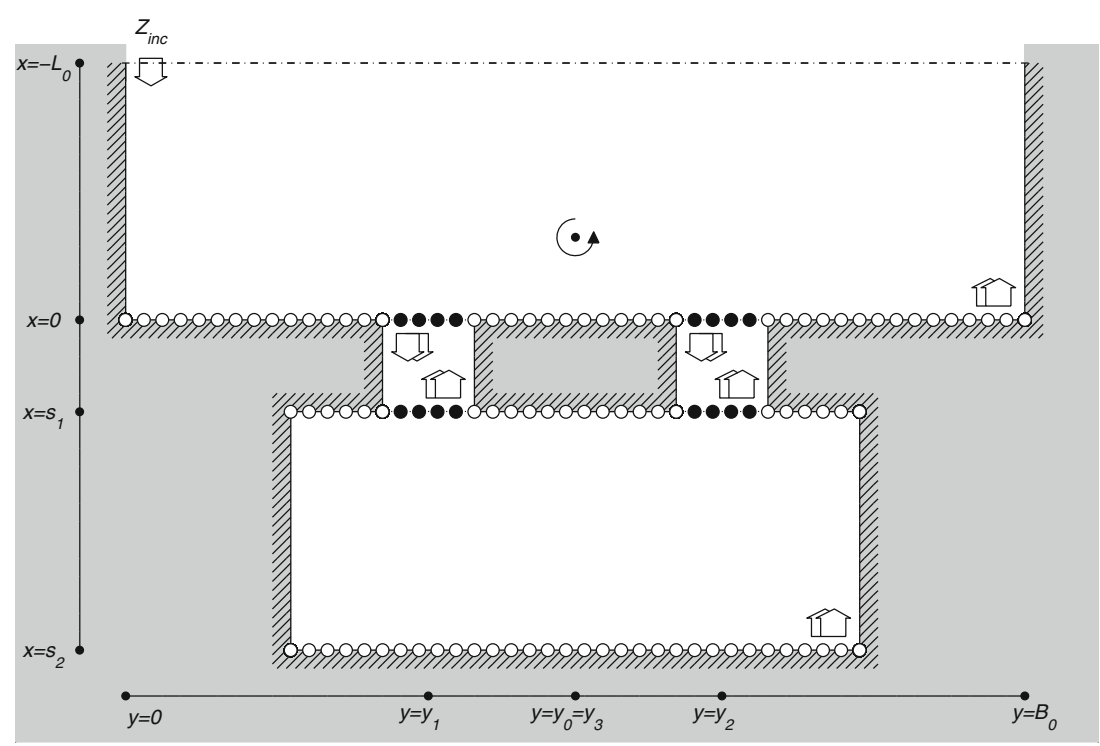


which is subsequently compared with $U_{k}^{\text {old }}$ and appropriately adjusted according to $U_{k}^{\text {new }}=U_{k}^{\text {old }}+\epsilon\left(U_{k}^{\text {new }}-U_{k}^{\text {old }}\right)$. Here, $\epsilon \sim 0.5$ is an under-relaxation factor that significantly accelerates the iteration process. The new (better) $U_{k}$ values and thus new $r_{k}$ values, in turn lead to a new solution and so forth. The iteration ends when the desired accuracy is obtained. Note that the feedback of the solution on the values of the friction coefficients is in fact a non-linear element in an otherwise linear hydrodynamic model.

\section{References}

Beets DJ, van der Valk L, Stive MJF (1992) Holocene evolution of the coast of Holland. Mar Geol 103(1-3):423-443. doi:10.1016/0025-3227(92)90030-L

Brouwer RL, van de Kreeke J, Schuttelaars HM (2012a) Entrance/exit losses and cross-sectional stability of double inlet systems. Est Coast Shelf Sci 107:69-80. doi:10.1016/j.ecss.2012.04.033

Brown EI (1928) Inlets on sandy coasts. Proc Am Soc Civ Eng 54(1):505-553

Bruun P, Mehta AJ, Johnsson IG (1978) Stability of tidal inlets: theory and engineering. Elsevier, The Netherlands, p 510

Buchwald VT (1971) The diffraction of tides by a narrow channel. J Fluid Mech 46(3):501-511. doi:10.1017/S0022112071000661

Dastgheib A, Roelvink JA, Wang ZB (2008) Long-term process-based morphological modeling of the Marsdiep Tidal Basin. Mar Geol 256(1-4):90-100. doi:10.1016/j.margeo.2008.10.003

Dronkers J (1998) Morphodynamics of the Dutch Delta. In: Dronkers J, Scheffers MBAM (eds) Physical estuar coastal seas: proceedings international conference. The Hague, pp 297-304

Elias EPL, Stive MJF, Bonekamp JG, Cleveringa J (2003) Tidal inlet dynamics in response to human intervention. Coast Eng $\mathrm{J}$ 45(4):629-658. doi:10.1142/S0578563403000932

Escoffier FF (1940) The stability of tidal inlets. Shore Beach 8(4):111114

Escoffier FF (1977) Hydraulics and stability of tidal inlets. GITI Report 13, US Army Coastal Engineering Research Center, Vicksburg

Garrett C (1975) Tides in gulfs. Deep-Sea Res 22(1):23-35. doi:10.1016/0011-7471(75)90015-7

Glaeser JD (1978) Global distribution of barrier island in terms of tectonic setting. J Geol 86(3):283-297

Godin G (1965) The M2 tide in the Labrador Sea, Davis Strait and Baffin Bay. Deep-Sea Res 12(4):469-477. doi:10.1016/00117471(65)90401-8

Jarrett JT (1976) Tidal prism-inlet area relationships. GITI Report 3. US Army Coastal Engineering Research Center, Vicksburg

van de Kreeke J (1985) Stability of tidal inlets-Pass Cavallo, Texas. Estuar Coast Shelf Sci 21(1):33-43. doi:10.1016/0272-7714(85) 90004-6

van de Kreeke J (1990a) Can multiple tidal inlets be stable? Estuar Coast Shelf Sci 30(3):261-273. doi:10.1016/02727714(90)90051-R

van de Kreeke J (1990b) Stability analysis of a two-inlet bay system. Coast Eng 14(6):481-497. doi:10.1016/0378-3839(90)90031-Q

van de Kreeke J (1992) Stability of tidal inlets; Escoffier's analysis. Shore and Beach 60:9-12

van de Kreeke J (1998) Adaptation of the Frisian Inlet to a reduction in basin area with special reference to the cross-sectional area. In: Dronkers J, Scheffers MBAM (eds) Physical estuar coastal seas: proceedings international conference. The Hague, pp 355362 van de Kreeke J (2004) Equilibrium and cross-sectional stability of tidal inlets: application to the Frisian Inlet before and after basin reduction. Coast Eng 51(5-6):337-350. doi:10.1016/ j.coastaleng.2004.05.002

van de Kreeke J, Brouwer RL, Zitman TJ, Schuttelaars HM (2008) The effect of a topographic high on the morphological stability of a two-inlet bay system. Coast Eng 55(4):319-332. doi:10.1016/ j.coastaleng.2007.11.010

LeConte LJ (1905) Discussion of Notes on the improvement of river and harbour outlets in the United States by DA Watts. T Am Soc Civ Eng LV(2):306-308

Lorentz HA (1926) Verslag Staatscommissie Zuiderzee 1918-1926. Den Haag, The Netherlands

Louters T, Gerritsen F (1994) The riddle of the sands; a tidal system's answer to a rising sea level. Report RIKZ-94.040, Nat Inst Coast Mar Manage, p 69

Maas LRM (1997) On the non-linear helmholtz response of almostenclosed tidal basins with sloping bottoms. J Fluid Mech 349:361380. doi:10.1017/S0022112097006824

Miles JW (1948) The coupling of a cylindrical tube to a half-infinite space. J Acoust Soc Am 20(5):652-664. doi:10.1121/1.1906423

National Park Service (2012) Post-hurricane Sandy: old inlet breach on Fire Island. http://www.nps.gov/fiis/naturescience/posthurricane-sandy-breaches.htm

O'Brien MP (1931) Estuary tidal prism related to entrance areas. Civ Eng 1(8):738-739

O'Brien MP, Dean RG (1972) Hydraulics and sedimentary stability of coastal inlets. In: 13th international conference coastal engineering, Vancouver, pp 761-780

Pacheco A, Vila-Concejo A, Ferreira Ó, Dias JA (2008) Assessment of tidal inlet evolution and stability using sediment budget computations and hydraulic parameter analysis. Mar Geol 247(12):104-127. doi:10.1016/j.margeo.2007.07.003

Pedlosky J (1987) Geophysical fluid dynamics. Springer, New York, p 728

Roos PC, Schuttelaars HM (2011) Influence of topography on tide propagation and amplification in semi-enclosed basins. Ocean Dyn 61(1):21-38. doi:10.1007/s10236-010-0340-0

Roos PC, Velema JJ, Hulscher SJMH, Stolk A (2011) An idealized model of tidal dynamics in the North Sea: resonance properties and response to large-scale changes. Ocean Dyn 61(12):2019-2035. doi:10.1007/s10236-011-0456-X

Roos PC, Schuttelaars HM, Brouwer RL (2013) Observations on barrier island length explained using an exploratory morphodynamic model. Geophys Res Lett 40. doi:10.1002/grl.50843

Salles P, Voulgaris G, Aubrey DG (2005) Contribution of nonlinear mechanisms in the persistence of multiple tidal inlet systems. Estuar Coast Shelf Sci 65(3):475-491. doi:10.1016/j.ecss. 2005.06.018

de Swart HE, Zimmerman JTF (2009) Morphodynamics of tidal inlet systems. Annu Rev Fluid Mech 41:203-229. doi:10.1146/ annurev.fluid.010908.165159

de Swart HE, Volp ND (2012) Effects of hypsometry on the morphodynamic stability of single and multiple tidal inlet systems. J Sea Res 74:35-44. doi:10.1016/j.seares.2012.05.008

Taylor GI (1920) Tidal oscillations in gulfs and rectangular basins. P Lond Math Soc s2-20(1):148-181. doi:10.1112/plms/s2-20.1. 148

Zimmerman JTF (1976) Mixing and flushing of tidal embayments in the western Dutch Wadden Sea part I: Distribution of salinity and calculation of mixing time scales. Neth J Sea Res 10(2):149-191. doi:10.1016/0077-7579(76)90013-2

Zimmerman JTF (1982) On the Lorentz linearization of a quadratically damped forced oscillator. Phys Lett A 89A(3):123-124. doi:10.1016/0375-9601(82)90871-4 